\title{
Microlocal Riemann-Hilbert Correspondence
}

\author{
By
}

Ingo WASCHKIES*

\begin{abstract}
We construct the global microlocal Riemann-Hilbert correspondence as an explicit equivalence between the abelian stack of microlocal perverse sheaves defined in $[\mathrm{W}]$ and the abelian stack of regular holonomic microdifferential modules of [KK]. The theory of analytic ind-sheaves and its microlocalization is crucial for our construction since it allows us to define solution complexes with values in the (ind-)ring of microlocal holomorphic functions (resp. microlocal tempered holomorphic functions).
\end{abstract}

\section{Contents}

$\S 1 . \quad$ Introduction

$\S 2$. Microlocal Perverse Sheaves and Contact Transformations

$\S 2.1$. Microlocal perverse sheaves

$\S 2.2$. Invariance under contact transformations

§2.3. Integral transforms for ind-sheaves

$\S 2.4$. Integral transforms for microlocal perverse sheaves

§2.5. Quantized contact transformations for microlocal perverse sheaves

$\S 3 . \quad$ Microlocal Riemann-Hilbert Correspondence

$\S 3.1$. The Ind-objects $\mu \mathcal{O}_{X}$ and $\mu \mathcal{O}_{X}^{t}$

§3.2. $\varepsilon_{X}$-modules

$\S 3.3$. The microdifferential structure of $\mu \mathcal{O}_{X}$ and $\mu \mathcal{O}_{X}^{t}$

Communicated by M. Kashiwara. Received September 12, 2003. Revised October 31, 2003.

2000 Mathematics Subject Classification(s): 35A27, 32C38, 32S60.

Key words: Sheaves, Perverse sheaves, Microlocal Sheaf Theory, Ind-sheaves, $\mathcal{D}$-modules, $\mathcal{E}$-modules, Riemann-Hilbert correspondence.

*Université Pierre et Marie Curie (Paris VI), 4, place Jussieu, 75252 Paris Cedex, France, e-mail: ingo@math.jussieu.fr 
§3.4. Quantized contact transformations for $\mu \mathcal{O}_{X}$ and $\mu \mathcal{O}_{X}^{t}$

§3.5. Classical Riemann-Hilbert Theorem and Ind-sheaves

§3.6. Microlocal Riemann-Hilbert morphism

A. Appendix - The Functor of Ind-microlocalization

$\S A .1 . \quad$ Ind-sheaves

$\S$ A.2. Microlocalization of ind-sheaves

References

\section{$\S 1$. Introduction}

In $[\mathrm{W}]$ we constructed for any field $k$ the abelian stack of microlocal perverse sheaves $\mu \operatorname{Perv}\left(k_{X}\right)$ on the projective cotangent bundle $P^{*} X$ of a complex manifold $X$. It is naturally embedded into the prestack of bounded derived categories of ind-sheaves on $\mathbb{C}^{\times}$-conic open subsets of $T^{*} X$. In the last section we announced that, over the field of complex numbers $\mathbb{C}$, our construction is equivalent to the stack of regular holonomic microdifferential modules by an explicit "microlocal Riemann-Hilbert correspondence".

Recall that using the ind-sheaf $\mathcal{O}^{t} \in \mathrm{D}^{\mathrm{b}}\left(\mathrm{I}\left(\mathbb{C}_{X}\right)\right)$ the classical RiemannHilbert correspondence can be expressed as the following diagram of quasiinverse contravariant equivalences between the category of perverse sheaves and the category of regular holonomic $\mathcal{D}_{X}$-modules (see $\left.[\mathrm{K} 2]\right)^{1}$

$$
\operatorname{Perv}\left(\mathbb{C}_{X}\right) \underset{\operatorname{RH} \mathcal{H} \operatorname{Hom}_{\mathcal{D}_{X}}\left(\cdot, \mathcal{O}_{X}\right)}{\stackrel{\left.\mathcal{O}^{t}\right)}{\rightleftarrows}} \mathcal{H o l} \operatorname{Reg}\left(\mathcal{D}_{X}\right) .
$$

Here the "ring" $\mathcal{O}^{t} \in \mathrm{D}^{\mathrm{b}}\left(\mathrm{I}\left(\mathbb{C}_{X}\right)\right)$ is the complex of tempered holomorphic functions in the bounded derived category of ind-sheaves on $X$ which has been constructed in [KS2] and $\mathrm{RH} \operatorname{Com}\left(\cdot, \mathcal{O}^{t}\right)$ is isomorphic to Kashiwara's functor of tempered solutions (see [K2] where it is denoted by THom). These equivalences can be extended to equivalences of abelian stacks on $X$.

The purpose of this paper is to establish the microlocal analogon in the form of quasi-inverse equivalences

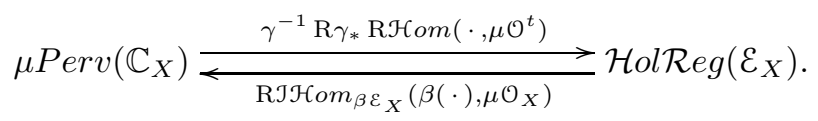

\footnotetext{
${ }^{1}$ Here and in the rest of this paper, we do not write the shift (by the dimension of the base manifold) that arises according to the choice of a definition of a perverse sheaf.
} 
Here $\gamma: T^{*} X \backslash T_{X}^{*} X \rightarrow P^{*} X$ is the natural projection, RJH Hom the derived functor of the interior Hom-functor for ind-sheaves, $\mu$ is Kashiwara's functor of ind-microlocalization (see [K3] and Appendix A) and $\beta$ is the embedding of sheaves into ind-sheaves that is used to transport the theory of modules over a sheaf of rings to ind-modules (see [KS2]).

If we look at the microlocal Riemann-Hilbert correspondence (1.0.1) in the stalks we find Andronikof's construction and the microlocal Riemann-Hilbert theorem announced in [An1]. Another construction of microlocal perverse sheaves and of a non-explicit microlocal Riemann-Hilbert correspondence was proposed in [GMV], but to our knowledge this project has neither been completed nor published.

The main idea to prove that the functors of (1.0.1) are well defined and indeed equivalences is the following. Both stacks $\mu \operatorname{Perv}\left(\mathbb{C}_{X}\right)$ and $\mathcal{H o l R e g}\left(\mathcal{E}_{X}\right)$ are locally invariant under quantized contact transformations. So the first task is to prove that the constructions of (1.0.1) are also invariant which allows us to reduce the situation to the so-called generic position. In generic position microlocal perverse sheaves come from perverse sheaves and regular holonomic microdifferential modules come from regular holonomic $\mathcal{D}$-modules. We will then show that in the generic position the functors (1.0.1) induce the classical Riemann-Hilbert correspondence which allows us to prove the theorem.

We would like to express our gratitude to Pierre Schapira who introduced us to the microlocal Riemann-Hilbert problem and who guided our work with invaluable help and encouragement during the last three years. We would also like to thank M. Kashiwara for his precious help and for giving us inside into his unpublished work on the microlocalization of ind-sheaves. Finally we thank F. Ivorra for many fruitful discussions during our effort to write a presentation of Kashiwara's work on ind-microlocalization.

\section{§2. Microlocal Perverse Sheaves and Contact Transformations}

\section{§2.1. Microlocal perverse sheaves}

In this section we briefly recall the construction of the abelian stack of microlocal perverse sheaves of $[\mathrm{W}]$ on the projective cotangent bundle $P^{*} X$ of a complex manifold $X$.

We denote by $\mathrm{D}^{\mathrm{b}}\left(k_{X}\right)$ the bounded derived category of sheaves of $k$ vector spaces on $X$ and by $\mathrm{D}_{\mathbb{R}-\mathrm{c}}^{\mathrm{b}}\left(k_{X}\right)$ the full subcategory of complexes with $\mathbb{R}$-constructible cohomology. We will not recall here the theory of the microsupport $\mathrm{SS}(\mathcal{F})$ of a sheaf $\mathcal{F}$ and refer to [KS1]. 
If $S \subset T^{*} X$ is a subset we consider the null system

$$
\mathcal{N}_{S}=\left\{\mathcal{F} \in \mathrm{D}^{\mathrm{b}}\left(k_{X}\right) \mid \mathrm{SS}(\mathcal{F}) \cap S=\varnothing\right\}
$$

and the microlocalizations

$$
\mathrm{D}^{\mathrm{b}}\left(k_{X}, S\right)=\mathrm{D}^{\mathrm{b}}\left(k_{X}\right) / \mathcal{N}_{S} \quad \text { and } \quad \mathrm{D}_{\mathbb{R}-\mathrm{c}}^{\mathrm{b}}\left(k_{X}, S\right)=\mathrm{D}_{\mathbb{R}-\mathrm{c}}^{\mathrm{b}}\left(k_{X}\right) / \mathcal{N}_{S} \cap \mathrm{D}_{\mathbb{R}-\mathrm{c}}^{\mathrm{b}}\left(k_{X}\right) .
$$

Definition 2.1.1. Let $S \subset \dot{T}^{*} X$ be a $\mathbb{C}^{\times}$-conic subset.

(i) An object $\mathcal{F} \in \mathrm{D}_{\mathbb{R}-\mathrm{c}}^{\mathrm{b}}\left(k_{X}\right)$ is called microlocally perverse on $S$, if there exists an open neighborhood $U$ of $S$ such that the micro-support $\operatorname{SS}(\mathcal{F})$ is $\mathbb{C}^{\times}$conic in $U^{2}$ and for every non-singular point $p$ of $\operatorname{SS}(\mathcal{F}) \cap U$ such that the projection $\pi: \mathrm{SS}(\mathcal{F}) \rightarrow X$ has constant rank in a neighborhood of $p$, there exists a submanifold $Y \subset X$ and an object $M \in \operatorname{Mod}(k)$ such that $\mathcal{F} \simeq M_{Y}[\operatorname{dim} Y]$ in $\mathrm{D}^{\mathrm{b}}\left(k_{X}, p\right)$.

(ii) We will denote by $\mathrm{D}_{\text {perv }}^{\mathrm{b}}\left(k_{X}, S\right)$ the full subcategory of $\mathrm{D}_{\mathbb{R}-\mathrm{c}}^{\mathrm{b}}\left(k_{X}, S\right)$ whose objects are perverse on $S$ (i.e. which may be represented by a microlocally perverse sheaf on $S$ ).

(iii) The prestack on $P^{*} X$

$$
\Omega \mapsto \mathrm{D}_{\text {perv }}^{b}\left(k_{X}, \gamma^{-1} \Omega\right)
$$

is called Andronikof's prestack of microlocal perverse sheaves.

An important property of Andronikof's prestack is its invariance under contact transformations (cf. [W]).

Proposition 2.1.2. Let $X, Y$ be two complex manifolds, $\Omega_{X} \subset \dot{T}^{*} X$, $\Omega_{Y} \subset \dot{T}^{*} Y$ open $\mathbb{C}^{\times}$-conic subsets and

$$
\chi: \Omega_{X} \stackrel{\sim}{\longrightarrow} \Omega_{Y}
$$

a contact transformation ${ }^{3}$. Let $\Lambda$ be the $\mathbb{C}^{\times}$-conic Lagrangian variety

$$
\Lambda=\left\{((y ; \eta),(x ; \xi)) \in \Omega_{Y} \times \Omega_{X}^{a} \mid(y, \eta)=\chi(x,-\xi)\right\} .
$$

Let $p_{X} \in \Omega_{X}$ and $p_{Y}=\chi\left(p_{X}\right)$.

There exist open neighborhoods $X^{\prime}$ of $\pi\left(p_{X}\right), Y^{\prime}$ of $\pi\left(p_{Y}\right), \mathbb{C}^{\times}$-conic open neighborhoods $\Omega_{X}^{\prime}$ of $\mathbb{C}^{\times} p_{X}, \Omega_{Y}^{\prime}$ of $\mathbb{C}^{\times} p_{Y}$ with $\Omega_{X}^{\prime} \subset T^{*} X^{\prime} \cap \Omega_{X}, \Omega_{Y}^{\prime} \subset T^{*} Y^{\prime} \cap$ $\Omega_{Y}$ and a kernel $\mathcal{K} \in \mathrm{D}_{\mathbb{R}-c}^{\mathrm{b}}\left(k_{Y^{\prime} \times X^{\prime}}\right)$ satisfying

\footnotetext{
${ }^{2}$ Such a sheaf is called microlocally $\mathbb{C}$-constructible on $U$.

${ }^{3}$ In this paper a contact transformation means an homogenous symplectic isomorphism.
} 
(1) $\chi$ induces a contact transformation $\Omega_{X}^{\prime} \stackrel{\sim}{\rightarrow} \Omega_{Y}^{\prime}$,

(2) $\quad\left(\left(\Omega_{Y}^{\prime \prime} \times T^{*} X^{\prime}\right) \cup\left(T^{*} Y^{\prime} \times \Omega_{X}^{\prime \prime}{ }^{a}\right)\right) \cap \operatorname{SS}(\mathcal{K}) \subset \Lambda \cap\left(\Omega_{Y}^{\prime \prime} \times \Omega_{X}^{\prime \prime}{ }^{a}\right)$ for every $\mathbb{C}^{\times}$-conic open subset $\Omega_{X}^{\prime \prime} \subset \Omega_{X}^{\prime}$ and $\Omega_{Y}^{\prime \prime}=\chi\left(\Omega_{X}^{\prime \prime}\right)$,

(3) the functor $\Phi_{\mathcal{K}}=\mathcal{K} \circ$ induces an equivalence of prestacks

$$
\Phi_{\mathcal{K}}^{\text {perv }}:\left.\left.\chi_{*} \mathrm{D}_{\text {perv }}^{\mathrm{b}}\left(k_{X^{\prime}}, *\right)\right|_{\Omega_{X}^{\prime}} \longrightarrow \mathrm{D}_{\text {perv }}^{\mathrm{b}}\left(k_{Y^{\prime}}, *\right)\right|_{\Omega_{Y}^{\prime}} .
$$

(4) $\operatorname{SS}\left(\Phi_{\mathcal{K}}^{\text {perv }}(\mathcal{F})\right) \cap \Omega_{Y}^{\prime \prime}=\chi\left(\operatorname{SS}(\mathcal{F}) \cap \Omega_{X}^{\prime \prime}\right)$

$\left.\left.(5) \chi_{*} \mu h o m(\mathcal{F}, \mathcal{G})\right|_{\Omega_{X}^{\prime}} \simeq \mu h o m\left(\Phi_{\mathcal{K}}^{\text {perv }}(\mathcal{F}), \Phi_{\mathcal{K}}^{\text {perv }}(\mathcal{G})\right)\right|_{\Omega_{Y}^{\prime}}$.

Note that if $\mathcal{F} \in \mathrm{D}_{\text {perv }}^{\mathrm{b}}\left(k_{X}, \mathbb{C}^{\times} p_{X}\right)$ then $\Phi_{\mathcal{K}}^{\text {perv }}(\mathcal{F}) \simeq \mathcal{K} \circ \mathcal{F}_{\bar{V}}$ for some subanalytic relatively compact open neighborhood $V$ of $\pi\left(p_{X}\right)$. It is not hard to calculate the stalks of Andronikof's prestack. We get (cf. [W])

Proposition 2.1.3. Let $p \in \dot{T}^{*} X$. The stalk of Andronikof's prestack of microlocal perverse sheaves at $\gamma(p)$ is naturally equivalent to the category $\mathrm{D}_{\text {perv }}^{b}\left(k_{X}, \mathbb{C}^{\times} p\right)$. It is an abelian category. Moreover the natural functor

$$
\mathrm{D}_{\text {perv }}^{\mathrm{b}}\left(k_{X}, \mathbb{C}^{\times} p\right) \longrightarrow \mathrm{D}^{\mathrm{b}}\left(k_{X}, \mathbb{C}^{\times} p\right)
$$

is fully faithful.

Recall (or see Appendix A) the functor of prestacks on $P^{*} X$

$$
\mu: \gamma_{*} \mathrm{D}_{\text {perv }}^{\mathrm{b}}\left(k_{X}, *\right) \longrightarrow \gamma_{*} \mathrm{D}^{\mathrm{b}}\left(\mathrm{I}\left(k_{*}\right)\right) .
$$

Next we define microlocal perverse sheaves as ind-sheaves on conic open subsets of $T^{*} X$.

Definition 2.1.4. Let $\Omega \subset P^{*} X$ be an open subset.

(1) An object $\mathcal{F} \in \mathrm{D}^{\mathrm{b}}\left(\mathrm{I}\left(k_{\gamma^{-1} \Omega}\right)\right)$ is microlocally perverse (on $\Omega$ ) if it is locally in the image of the functor (2.1.1), i.e. if for all $p \in \gamma^{-1}(\Omega)$ there exist a $\mathbb{C}^{\times}$-conic neighborhood $V \supset \mathbb{C}^{\times} p$ and an object $\mathcal{G} \in \mathrm{D}_{\text {perv }}^{\mathrm{b}}\left(k_{X}, V\right)$ such that $\left.\left.\mu \mathcal{G}\right|_{V} \simeq \mathcal{F}\right|_{V}$.

(2) We denote by $\mu \operatorname{Perv}(\Omega)$ the full subcategory of $\mathrm{D}^{\mathrm{b}}\left(\mathrm{I}\left(k_{\gamma^{-1} \Omega}\right)\right)$ whose objects are microlocally perverse. 
The main result of $[\mathrm{W}]$ is

Theorem 2.1.5. The prestack $\mu$ Perv on $P^{*} X$ is an abelian stack. Moreover the functor $\mu$ induces an equivalence of abelian stacks

$$
\mathrm{D}_{\text {perv }}^{\mathrm{b}}\left(k_{X}, *\right)^{\ddagger} \stackrel{\sim}{\longrightarrow} \mu \text { Perv },
$$

where $\mathrm{D}_{\text {perv }}^{\mathrm{b}}\left(k_{X}, *\right)^{\ddagger}$ denotes the stack associated to Andronikof's prestack.

The support of a microlocal perverse sheaf is by construction a homogenous Lagrangian subvariety. As in the case of microdifferential modules we are able to characterize microlocal perverse sheaves in generic position ${ }^{4}$.

Proposition 2.1.6. $\quad$ Let $\Lambda$ be a homogenous Lagrangian variety and suppose that $\Lambda$ is in generic position at $p \in \dot{T}^{*} X$. Then $\mu$ induces a fully faithful functor

$$
\operatorname{Perv}_{\pi(p), \Lambda} \longrightarrow \mu \operatorname{Perv}_{\gamma(p)},
$$

where $\operatorname{Perv}_{\pi(p), \Lambda}$ is the category germs of perverse sheaves at $\pi(p)$ (modulo constant sheaves) whose micro-support is included in $\Lambda$ in a neighborhood of $\gamma(p)$. Its image are all microlocal perverse sheaves whose support is included in $\Lambda$ (in a neighborhood of $\gamma(p))$.

\section{§2.2. Invariance under contact transformations}

Let $p \in \Omega_{X} \subset T^{*} X, q \in \Omega_{Y} \subset T^{*} Y$ where $\Omega_{X}, \Omega_{Y}$ are $\mathbb{C}^{\times}$-conic open subsets. Suppose that we are given a contact transformation

$$
\chi: \Omega_{X} \stackrel{\sim}{\longrightarrow} \Omega_{Y}
$$

such that $\chi(p)=q$. After shrinking $\Omega_{X}$ and $\Omega_{Y}$, we can establish an equivalence of prestacks (cf. Proposition 2.1.2)

$$
\Phi_{\mathcal{K}}^{\text {perv }}=\mathcal{K}_{\circ}:\left.\left.\chi_{*} \mathrm{D}_{\text {perv }}^{\mathrm{b}}\left(k_{X}, *\right)\right|_{\Omega_{X}} \stackrel{\sim}{\longrightarrow} \mathrm{D}_{\text {perv }}^{\mathrm{b}}\left(k_{Y}, *\right)\right|_{\Omega_{Y}} .
$$

Hence we get an equivalence of the associated stacks of microlocal perverse sheaves:

$$
\left.\left.\chi_{*} \mu \operatorname{Perv}{ }^{X}\right|_{\gamma\left(\Omega_{X}\right)} \stackrel{\sim}{\longrightarrow} \mu \operatorname{Perv}^{Y}\right|_{\gamma\left(\Omega_{Y}\right)}
$$

\footnotetext{
${ }^{4} \mathrm{~A} \mathbb{C}^{\times}$-homogenous Lagrangian variety is said to be in generic position at $p \in \dot{T}^{*} X$ if locally at $p$ we have $\Lambda \cap \pi^{-1} \pi(p) \subset \mathbb{C}^{\times} p$.
} 
together with a commutative diagram (up to isomorphism)

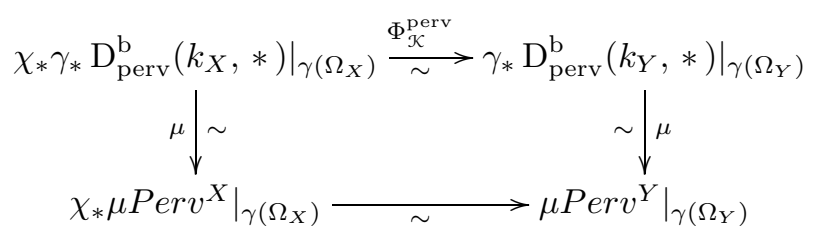

The existence of this diagram is sufficient for many applications. However, for concrete calculations we need to know that the equivalence (2.2.1) is explicitly given by integral transform with the kernel $\mu \mathcal{K}$ which we will prove in Section 1.5 .

\section{§2.3. Integral transforms for ind-sheaves}

Let us fix some notations. We will first consider real manifolds $X, Y, Z$ and the diagram

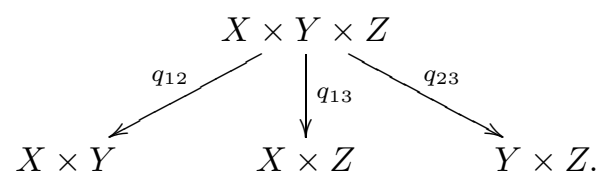

Composition (or integral transforms) will be considered in its ind-version. For $\mathcal{F} \in \mathrm{D}^{\mathrm{b}}\left(\mathrm{I}\left(k_{X \times Y}\right)\right)$ and $\mathcal{G} \in \mathrm{D}^{\mathrm{b}}\left(\mathrm{I}\left(k_{Y \times Z}\right)\right)$ we set

$$
\mathcal{F} \circ \mathcal{G}=\mathrm{R} q_{13 ! !}\left(q_{12}^{-1} \mathcal{F} \otimes q_{23}^{-1} \mathcal{G}\right)
$$

even if $\mathcal{F}, \mathcal{G}$ are actually complexes of classical sheaves. In this case $\alpha(\mathcal{F} \circ \mathcal{G})$ gives the classical composition. Note that there is always a natural morphism

$$
\mathcal{F} \circ \mathcal{G} \longrightarrow \alpha(\mathcal{F} \circ \mathcal{G})
$$

Furthermore, we will consider the following variant. Let

$$
\begin{aligned}
q_{12}^{a}: T^{*} X \times T^{*} Y \times T^{*} Z & \longrightarrow T^{*} X \times T^{*} Y \\
((x ; \xi),(y ; \eta),(z ; \zeta)) & \mapsto((x ; \xi),(y,-\eta)) .
\end{aligned}
$$

Then set for $\mathcal{F} \in \mathrm{D}^{\mathrm{b}}\left(\mathrm{I}\left(k_{T^{*} X \times T^{*} Y}\right)\right)$ and $\mathcal{G} \in \mathrm{D}^{\mathrm{b}}\left(\mathrm{I}\left(k_{T^{*} Y \times T^{*} Z}\right)\right)$

$$
\mathcal{F} \stackrel{a}{\circ} \mathcal{G}=\operatorname{R} q_{13 ! !}\left(q_{12}^{a-1} \mathcal{F} \otimes q_{23}^{-1} \mathcal{G}\right) .
$$

Note that the operation $\stackrel{a}{\circ}$ is associative up to natural isomorphism. 


\section{§2.4. Integral transforms for microlocal perverse sheaves}

For any kernel $\mathcal{K} \in \mathrm{D}^{\mathrm{b}}\left(k_{Y \times X}\right)$ attached to contact transformations $\chi$ : $\Omega_{X} \stackrel{\sim}{\longrightarrow} \Omega_{Y}$ we study the functor

$$
\Phi_{\mu \mathcal{K}}^{a}=\mu \mathcal{K}_{\circ}^{a}:\left.\left.\chi_{*} \gamma_{*} \mathrm{D}^{\mathrm{b}}\left(\mathrm{I}\left(k_{*}\right)\right)\right|_{\gamma\left(\Omega_{X}\right)} \longrightarrow \gamma_{*} \mathrm{D}^{\mathrm{b}}\left(\mathrm{I}\left(k_{*}\right)\right)\right|_{\gamma\left(\Omega_{Y}\right)} .
$$

Our interest in this section is to find kernels $\mathcal{K}$ such that this functor preserves the stack $\mu$ Perv of microlocal perverse sheaves.

By definition an object in $\gamma_{*} \mathrm{D}^{\mathrm{b}}\left(\mathrm{I}\left(k_{*}\right)\right)$ is microlocally perverse on $\Omega_{Y}$ if and only if it is microlocally perverse in the stalks, i.e. in the category $\gamma_{*} \mathrm{D}^{\mathrm{b}}\left(\mathrm{I}\left(k_{*}\right)\right)_{\gamma(p)}$ for every $p \in \Omega_{Y}$.

Take an object $\mathcal{F} \in \mu$ Perv. By definition we have locally $\mathcal{F} \simeq \mu \widetilde{\mathcal{F}}$ for some object $\widetilde{\mathcal{F}} \in \mathrm{D}_{\text {perv }}^{\mathrm{b}}\left(k_{X}, \mathbb{C}^{\times} p\right)$. Hence locally

$$
\mu \mathcal{K} \stackrel{a}{\circ} \mathcal{F} \simeq \mu \mathcal{K}^{a} \circ \mu \widetilde{\mathcal{F}} .
$$

Unfortunately, the functor $\mu$ commutes with $\stackrel{a}{\circ}$ only in special situations (see Appendix A, Theorem A.2.5), and therefore we will have to find kernels $\mathcal{K}$ such that

$$
\mu \mathcal{K} \stackrel{a}{\circ} \mu \widetilde{\mathcal{F}} \simeq \mu(\mathcal{K} \circ \widetilde{\mathcal{F}})
$$

holds in a neighborhood of $\mathbb{C}^{\times} q$. This would imply that $\Phi_{\mu \mathcal{K}}^{a}$ induces locally

$$
\Phi_{\mu \mathcal{K}}^{a}:\left.\left.\chi_{*} \mu \operatorname{Perv}\left(k_{X}\right)\right|_{\gamma\left(\Omega_{X}\right)} \longrightarrow \mu \operatorname{Perv}\left(k_{Y}\right)\right|_{\gamma\left(\Omega_{Y}\right)} .
$$

By Theorem A.2.5 there is at least a natural morphism

$$
\mu \mathcal{K} \stackrel{a}{\circ} \mu \widetilde{\mathcal{F}} \longrightarrow \mu(\mathcal{K} \circ \widetilde{\mathcal{F}}) .
$$

Remark 2.4.1. Suppose condition (1) or (2) below.

(1) $\left(T_{X}^{*} X \times T^{*} Y \cup T^{*} X \times T_{Y}^{*} Y\right) \cap \operatorname{SS}\left(\mathcal{K}_{1}\right) \subset T_{X}^{*} X \times T_{Y}^{*} Y$,

(2) $\left(T_{Y}^{*} Y \times T^{*} Z \cup T^{*} Y \times T_{Z}^{*} Z\right) \cap \operatorname{SS}\left(\mathcal{K}_{2}\right) \subset T_{Y}^{*} Y \times T_{Z}^{*} Z$.

Then the natural morphism (2.4.1) is an isomorphism outside the zero section.

In particular, let $\mathcal{F} \in \mathrm{D}^{\mathrm{b}}\left(\mathrm{I}\left(k_{X}\right)\right)$ and consider $\mathcal{K}$ such that

$$
\operatorname{SS}(\mathcal{K}) \cap\left(T_{Y}^{*} Y \times T^{*} X \cup T^{*} Y \cup T_{X}^{*} X\right) \subset T_{Y}^{*} Y \times T_{X}^{*} X
$$

Then the morphism (2.4.1)

$$
\mu(\mathcal{K}) \stackrel{a}{\circ} \mu \mathcal{F} \longrightarrow \mu(\mathcal{K} \circ \mathcal{F})
$$

is an isomorphism outside the zero section. For instance we get the following lemma. 
Lemma 2.4.2. Let $\mathcal{F} \in \mathrm{D}^{\mathrm{b}}\left(\mathrm{I}\left(k_{X}\right)\right)$. The morphism

$$
\mu\left(k_{\Delta}\right) \stackrel{a}{\circ} \mu \mathcal{F} \longrightarrow \mu\left(k_{\Delta} \circ \mathcal{F}\right) \simeq \mu \mathcal{F}
$$

is an isomorphism outside the zero section.

We will mostly be interested in the following more general situation:

Definition 2.4.3. Let us denote by $\widetilde{\mathrm{N}}\left(Y, X, \Omega_{Y}, \Omega_{X}\right)$ the full subcategory of $\mathrm{D}^{\mathrm{b}}\left(k_{Y \times X}, \Omega_{Y} \times T^{*} X\right)$ such that

(i) $\operatorname{SS}(\mathcal{K}) \cap\left(\Omega_{Y} \times T^{*} X \cup T^{*} Y \times \Omega_{X}^{a}\right) \subset \Omega_{Y} \times \Omega_{X}^{a}{ }^{5}$

(ii) $p_{1}: \operatorname{SS}(\mathcal{K}) \cap \Omega_{Y} \times T^{*} X \rightarrow \Omega_{Y}$ is proper.

Proposition 2.4.4. Suppose that $\Omega_{X} \cap T_{X}^{*} X=\varnothing$ and $\Omega_{Y} \cap T_{Y}^{*} Y=\varnothing$. Let $\mathcal{K} \in \widetilde{\mathrm{N}}\left(Y, X, \Omega_{Y}, \Omega_{X}\right)$ and $\mathcal{F} \in \mathrm{D}^{\mathrm{b}}\left(k_{X}\right)$ such that $\operatorname{SS}(\mathcal{F}) \cap \dot{T}^{*} X \subset \Omega_{X}$. Then the natural morphism

$$
\mu \mathcal{K}^{a} \mu \mathcal{F} \longrightarrow \mu(\mathcal{K} \circ \mathcal{F})
$$

is an isomorphism on $\Omega_{Y}$.

Proof. We have by hypothesis

$$
\begin{aligned}
\operatorname{SS}(\mathcal{K}) \cap\left(T^{*} Y \times \operatorname{SS}(\mathcal{F})^{a}\right) & \subset\left(\operatorname{SS}(\mathcal{K}) \cap T^{*} Y \times \Omega_{X}^{a}\right) \cup\left(\operatorname{SS}(\mathcal{K}) \cap T^{*} Y \times T_{X}^{*} X\right) \\
& \subset \Omega_{Y} \times \Omega_{X}^{a} \cup T^{*} Y \times T_{X}^{*} X .
\end{aligned}
$$

Intersecting both sides with $T_{Y}^{*} Y \times T^{*} X$ we get

$$
\mathrm{SS}(\mathcal{K}) \cap\left(T^{*} Y \times \operatorname{SS}(\mathcal{F})^{a}\right) \cap\left(T_{Y}^{*} Y \times T^{*} X\right) \subset T_{X}^{*} X \times T_{Y}^{*} Y .
$$

Hence the non-characteristic condition of Theorem A.2.5 is satisfied. Moreover by assumption $\operatorname{SS}(\mathcal{K}) \cap\left(\Omega_{Y} \times T^{*} X\right) \subset \Omega_{Y} \times \Omega_{X}^{a}$, hence

$$
\mathrm{SS}(\mathcal{K}) \cap\left(T^{*} Y \times \operatorname{SS}(\mathcal{F})^{a}\right) \cap \Omega_{Y} \times T_{X}^{*} X=\varnothing
$$

and

$$
\mathrm{SS}(\mathcal{K}) \cap\left(T^{*} Y \times \mathrm{SS}(\mathcal{F})^{a}\right) \cap T^{*} Y \times T_{X}^{*} X \subset \complement \Omega_{Y} \times T_{X}^{*} X
$$

Therefore the morphism is an isomorphism outside $\complement \Omega_{Y}$, hence on $\Omega_{Y}$.

\footnotetext{
${ }^{5}$ Here, $\Omega^{a}$ denotes the image of $\Omega$ by the antipodal map.
} 
Remark 2.4.5. Consider $\mathcal{K} \in \widetilde{\mathrm{N}}\left(Y, X, \Omega_{Y}, \Omega_{X}\right)$ and $\mathcal{F} \in \mathrm{D}^{\mathrm{b}}\left(k_{X}\right)$. Then we have a chain of natural isomorphisms:

$$
\left.\left.\left.\left.\left.(\mu \mathcal{K})\right|_{\Omega_{Y} \times \Omega_{X}^{a}} \stackrel{a}{\circ}(\mu \mathcal{F})\right|_{\Omega_{X}} \simeq(\mu \mathcal{K})^{(1, a)}\right|_{\Omega_{Y} \times \Omega_{X}} \circ(\mu \mathcal{F})\right|_{\Omega_{X}} \simeq(\mu \mathcal{K} \stackrel{a}{\circ} \mu \mathcal{F})\right|_{\Omega_{Y}} .
$$

which is easily seen using the fact that $\operatorname{SS}(\mathcal{K})=\operatorname{supp}(\mu \mathcal{K})$. Consider the morphism

$$
\left.\left.\left.(\mu \mathcal{K} \stackrel{a}{\circ} \mu \mathcal{F})\right|_{\Omega_{Y}} \longrightarrow \mu(\mathcal{K} \circ \mathcal{F})\right|_{\Omega_{Y}} \longrightarrow \mu(\alpha(\mathcal{K} \circ \mathcal{F}))\right|_{\Omega_{Y}}
$$

The term on the right only depends on the image of $\mathcal{F}$ in $\mathrm{D}^{\mathrm{b}}\left(k_{X}, \Omega_{X}\right)$. By the last lemma, the same result holds for the term on the left side. In the following we will be interested in situations when the composition (2.4.2) is an isomorphism.

Hence, in the situation of Proposition 2.4.4, we may replace $\mathcal{F}$ by any object isomorphic to $\mathcal{F}$ in $\mathrm{D}^{\mathrm{b}}\left(k_{X}, \Omega_{X}\right)$. However, in general, it is not possible to find an object $\mathcal{F}^{\prime}$ isomorphic to $\mathcal{F}$ in $\mathrm{D}^{\mathrm{b}}\left(k_{X}, \Omega_{X}\right)$ such that $\operatorname{SS}\left(\mathcal{F}^{\prime}\right) \subset \Omega_{X}$. Therefore we will have to assume a stability condition on $\mathcal{K}$ and the existence of a suitable cut-off functor.

Proposition 2.4.6. Let $X, Y$ be affine and $\mathcal{K} \in \widetilde{\mathrm{N}}\left(Y, X, \Omega_{Y}, \Omega_{X}\right)$. Let $\delta \subset X^{*}$ be an open cone and $U \subset X$ be a relatively compact open set such that

$$
(\bar{U} \times \bar{\delta}) \cap \dot{T}^{*} X \subset \Omega_{X} .
$$

Set $\Omega_{X}^{\prime}=U \times \delta$ and suppose that there exists an open subset $\Omega_{Y}^{\prime} \subset \Omega_{Y}$ such that $\mathcal{K} \in \widetilde{\mathrm{N}}\left(Y, X, \Omega_{Y}^{\prime}, \Omega_{X}^{\prime}\right)$. Then the natural morphism

$$
\mu \mathcal{K} \stackrel{a}{\circ} \mu \mathcal{F} \longrightarrow \mu(\alpha(\mathcal{K} \circ \mathcal{F}))
$$

is an isomorphism on $\Omega_{Y}^{\prime}$.

Proof. There is a cut-off functor $\Phi_{U, \delta}$ (cf. [W]) such that $\operatorname{SS}\left(\Phi_{U, \delta}(\mathcal{F})\right) \subset$ $\bar{U} \times \bar{\delta}$ and $\Phi_{U, \delta}(\mathcal{F}) \rightarrow \mathcal{F}$ is an isomorphism in $\mathrm{D}^{\mathrm{b}}\left(k_{X}, U \times \delta\right)$. Then the morphism

$$
\mu \mathcal{K}^{a} \circ \mu\left(\Phi_{U, \delta}(\mathcal{F})\right) \longrightarrow \mu\left(\mathcal{K} \circ \Phi_{U, \delta}(\mathcal{F})\right)
$$

is an isomorphism on $\Omega_{Y}^{\prime}$. Moreover $\operatorname{supp}\left(\Phi_{U, \gamma}(\mathcal{F})\right) \subset \bar{U}$ which is compact. Hence $\mathcal{K} \circ \Phi_{U, \gamma}(\mathcal{F}) \rightarrow \alpha\left(\mathcal{K} \circ \Phi_{U, \gamma}(\mathcal{F})\right)$ is an isomorphism. Therefore

$$
\mu \mathcal{K}^{a} \circ \mu\left(\Phi_{U, \delta}(\mathcal{F})\right) \longrightarrow \mu\left(\alpha\left(\mathcal{K} \circ \Phi_{U, \delta}(\mathcal{F})\right)\right)
$$

is an isomorphism on $\Omega_{Y}^{\prime}$ and is isomorphic to

$$
\mu \mathcal{K} \stackrel{a}{\circ} \mu \mathcal{F} \longrightarrow \mu(\alpha(\mathcal{K} \circ \mathcal{F})) .
$$




\section{$\S 2.5$. Quantized contact transformations for microlocal perverse sheaves}

We are now ready to prove the main result of the first Section:

Theorem 2.5.1. Let $p \in \Omega_{X} \subset T^{*} X, q \in \Omega_{Y} \subset T^{*} Y$ where $\Omega_{X}, \Omega_{Y}$ are $\mathbb{C}^{\times}$-conic open subsets. Suppose that we are given a contact transformation

$$
\chi: \Omega_{X} \stackrel{\sim}{\longrightarrow} \Omega_{Y}
$$

such that $\chi(p)=q$. Then locally there exists a kernel $\mathcal{K}$ such that

$$
\mu \mathcal{K}_{\circ}^{a}:\left.\left.\chi_{*} \gamma_{*} \mathrm{D}^{\mathrm{b}}\left(\mathrm{I}\left(k_{*}\right)\right)\right|_{\gamma\left(\Omega_{X}\right)} \longrightarrow \gamma_{*} \mathrm{D}^{\mathrm{b}}\left(\mathrm{I}\left(k_{*}\right)\right)\right|_{\gamma\left(\Omega_{Y}\right)}
$$

induces an equivalence of stacks

$$
\left.\left.\chi_{*} \mu \operatorname{Perv}^{X}\right|_{\gamma\left(\Omega_{X}\right)} \stackrel{\sim}{\longrightarrow} \mu \operatorname{Perv}{ }^{Y}\right|_{\gamma\left(\Omega_{Y}\right)}
$$

and the diagram

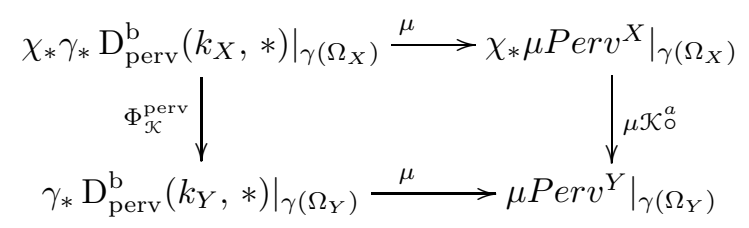

commutes up to natural isomorphism.

Proof. Consider the diagram of functors of prestacks

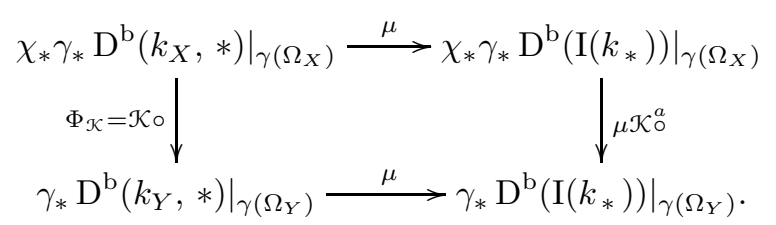

By Proposition 2.4.6 this diagram induces a commutative diagram (up to natural isomorphism) in the stalks, so we immediately get that for $p \in \Omega_{X}$ and 
$q=\chi(p) \in \Omega_{Y}$ the diagram

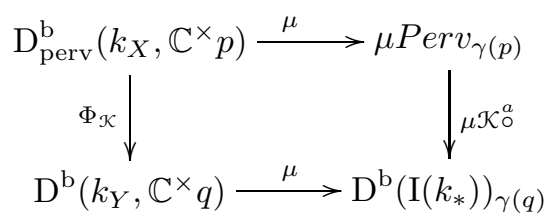

commutes up to natural isomorphism.

Consider the diagram (2.5.1). Let $\mathcal{F} \in \mathrm{D}_{\text {perv }}^{\mathrm{b}}\left(k_{X}, \mathbb{C}^{\times} p\right)$. Recall that for a sufficiently small relatively compact open neighborhood $V$ of $\pi(p)$ the object $\Phi_{\mathcal{K}}^{\text {perv }} \mathcal{F}$ is represented by $\mathcal{K} \circ \mathcal{F}_{\bar{V}}$ in $D_{\text {perv }}^{\text {b }}\left(k_{Y}, \mathbb{C}^{\times} q\right)$ and it is isomorphic to $\mathcal{K} \circ \mathcal{F}$ in $\mathrm{D}^{\mathrm{b}}\left(k_{Y}, \mathbb{C}^{\times} q\right)$. Since $\mathrm{D}_{\text {perv }}^{\mathrm{b}}\left(k_{Y}, \mathbb{C}^{\times} q\right)$ is a full subcategory of $\mathrm{D}^{\mathrm{b}}\left(k_{Y}, \mathbb{C}^{\times} q\right)$, we get that $\mu \mathcal{K}^{a} \stackrel{\sim}{\circ} \mathcal{F}$ is an object of $\mu \operatorname{Per} v_{\gamma(q)}^{Y}$. Hence we get the induced functor of stacks

$$
\left.\left.\mu \operatorname{Perv}{ }^{X}\right|_{\gamma\left(\Omega_{X}\right)} \longrightarrow \mu \operatorname{Perv}{ }^{Y}\right|_{\gamma\left(\Omega_{Y}\right)}
$$

and the diagram of the theorem.

In the same way we verify that $\mu \mathcal{K}^{*}{ }_{\circ}^{a}$ is a well defined functor of stacks in the opposite direction and it is easily verified that $\mu \mathcal{K}^{*} \circ$ is a quasi-inverse (as $\Phi_{\mathcal{K}^{*}}^{\text {perv }}$ is a quasi-inverse to $\Phi_{\mathcal{K}}^{\text {perv }}$ ).

Finally let us compose quantized contact transformations. Suppose that we have contact transformations

$$
\chi_{1}: \Omega_{X} \stackrel{\sim}{\rightarrow} \Omega_{Y} \quad \chi_{2}: \Omega_{Y} \stackrel{\sim}{\rightarrow} \Omega_{Z}
$$

and kernels $\mathcal{K}_{1} \in \mathrm{D}_{\mathbb{R}-\mathrm{c}}^{\mathrm{b}}\left(k_{Y \times X}\right), \mathcal{K}_{2} \in \mathrm{D}_{\mathbb{R}-\mathrm{c}}^{\mathrm{b}}\left(k_{Z \times Y}\right)$ such that Theorem 2.1.2 is valid. Then we know that $\Phi_{\mu \mathcal{K}_{1}}^{a}=\mu \mathcal{K}_{1}{ }^{a}$ and $\Phi_{\mu \mathcal{K}_{2}}^{a}=\mu \mathcal{K}_{2}{ }^{a}$ are well-defined on microlocal perverse sheaves. However note that $\mathcal{K}_{2} \circ \mathcal{K}_{1}$ is not $\mathbb{R}$-constructible and we do not know if

$$
\mu \mathcal{K}_{2} \stackrel{a}{\circ} \mu \mathcal{K}_{1} \longrightarrow \mu\left(\mathcal{K}_{2} \circ \mathcal{K}_{1}\right)
$$

is an isomorphism in $\mathrm{D}^{\mathrm{b}}\left(\mathrm{I}\left(k_{T^{*} Z \times T^{*} X}\right)\right)$. Nevertheless we get

Proposition 2.5.2. The functor

$$
\mu\left(\mathcal{K}_{2} \circ \mathcal{K}_{1}\right)^{a}: \mu \operatorname{Perv}^{X} \longrightarrow \mu \operatorname{Perv}{ }^{Z}
$$

is well-defined and naturally isomorphic to $\Phi_{\mu \mathcal{K}_{1}}^{a} \circ \Phi_{\mu \mathcal{K}_{1}}^{a}$.

\footnotetext{
${ }^{6}$ Here $\mathcal{K}^{*}=\operatorname{R} \mathcal{H o m}\left(\mathcal{K}, \omega_{X \times Y \mid X}\right)$
} 
Proof. The strategy is similar to the proof of Theorem 2.5.1. First we can show that $\mu\left(\mathcal{K}_{2} \circ \mathcal{K}_{1}\right)^{a}: \mu \operatorname{Perv}^{X} \longrightarrow \mu$ Perv ${ }^{Z}$ is well-defined by looking in the stalks and using the fact that $\mathrm{D}_{\text {perv }}^{\mathrm{b}}\left(k_{Y}, \mathbb{C}^{\times} q\right)$ is a full subcategory of $\mathrm{D}^{\mathrm{b}}\left(k_{Y}, \mathbb{C}^{\times} q\right)$. The same argument shows the isomorphism.

Remark 2.5.3. Theorem 2.5.1 and Proposition 2.5.2 are an important step towards the definition of the stack of microlocal perverse sheaves on a complex contact manifold. Such a manifold is locally isomorphic to an open subset $\Omega_{X} \subset P^{*} X$ and the transition maps are contact transformations. Using the proposition we can locally associate an equivalence of stacks. But the choice of the kernel $\mathcal{K}$ is neither unique nor canonical.

\section{§3. Microlocal Riemann-Hilbert Correspondence}

The classical Riemann-Hilbert correspondence states that on a complex variety $X$ the solution functor $\mathrm{R} \mathcal{H} \operatorname{Hom}_{\mathcal{D}_{X}}\left(\cdot, \mathcal{O}_{X}\right)$ defines a contravariant equivalence between the stack $\mathcal{H}$ olReg $\left(\mathcal{D}_{X}\right)$ of regular holonomic $\mathcal{D}_{X}$-modules and the stack of perverse sheaves. A quasi-inverse of this functor, $\operatorname{THom}\left(\cdot, \mathcal{O}_{X}\right)$, has been constructed explicitly by Kashiwara (cf. [K2]).

Let $\mathcal{M}$ be a holonomic $\mathcal{D}$-module. We set

$$
\operatorname{Sol}(\mathcal{M})=\operatorname{R} \mathcal{H} \operatorname{om}_{\mathcal{D}_{X}}\left(\mathcal{M}, \mathcal{O}_{X}\right)
$$

Let $\mathcal{F}$ be an object of $\mathrm{D}_{\mathbb{R}-\mathrm{c}}^{\mathrm{b}}\left(\mathbb{C}_{X}\right)$ and set

$$
\operatorname{RH}(\mathcal{F})=\operatorname{TH} \mathcal{H o m}\left(\mathcal{F}, \mathcal{O}_{X}\right)
$$

Then the Riemann-Hilbert correspondence can be stated as follows.

Theorem 3.0.4. The functors $\mathrm{RH}$ and Sol define quasi-inverse equivalences of abelian stacks

$$
\operatorname{Perv}\left(\mathbb{C}_{X}\right) \underset{\text { Sol }}{\stackrel{\mathrm{RH}}{\rightleftarrows}} \mathcal{H o l} \operatorname{Reg}\left(\mathcal{D}_{X}\right) .
$$

The microlocal Riemann-Hilbert correspondence should therefore establish an equivalence between the stack of microlocal perverse sheaves and the stack of regular holonomic $\mathcal{E}_{X}$-modules. 


\section{$\S 3.1$. The Ind-objects $\mu \mathcal{O}_{X}$ and $\mu \mathcal{O}_{X}^{t}$}

The microlocalization of the ring of holomorphic functions defines an object of $\mathrm{I}\left(\mathbb{C}_{X}\right)$. More precisely, we have (cf. [K3])

Proposition 3.1.1. The ind-sheaf

$$
\left.\mu \mathcal{O}_{X}\right|_{\dot{T}^{*} X}
$$

is concentrated in degree $d_{X}$.

Let $\mathcal{F} \in \mathrm{D}_{\mathbb{R}-\mathrm{c}}^{\mathrm{b}}\left(\mathbb{C}_{X}\right)$. We will study the microlocal solution complex

$$
\operatorname{\mu hom}\left(\mathcal{F}, \mathcal{O}_{X}\right) \simeq \operatorname{RH} \mathcal{H} o m\left(\pi^{-1} \mathcal{F}, \mu \mathcal{O}_{X}\right) .
$$

The stalks of this complex have been studied in [KS1] and we will show that some results can be extended to open neighborhoods.

The "ring" $\mathcal{O}_{X}^{t} \in \mathrm{D}^{\mathrm{b}}\left(\mathrm{I}\left(\mathbb{C}_{X}\right)\right)$ of temperate holomorphic functions has been defined in $[\mathrm{KS} 2]$. It is defined from the "ring" $\mathcal{D} b_{X}^{t} \in \mathrm{D}^{\mathrm{b}}\left(\mathrm{I}\left(\mathbb{C}_{X}\right)\right)$ as

$$
\mathcal{O}_{X}^{t}=\mathrm{RH}_{\mathcal{H}} \operatorname{mom}_{\beta\left(\mathcal{D}_{\bar{X}}\right)}\left(\beta\left(\mathcal{O}_{\bar{X}}\right), \mathcal{D} b_{X}^{t}\right) .
$$

We will not recall the construction of $\mathcal{D} b_{X}^{t}$ here. Recall that $\mathcal{O}_{X}^{t}$ is only defined in the derived category. It is not concentrated in a single degree.

The link with Kashiwara's functor THom is given by the natural isomorphism

$$
\operatorname{R\mathcal {H}om}\left(\mathcal{F}, \mathcal{O}_{X}^{t}\right) \simeq \operatorname{THom}\left(\mathcal{F}, \mathcal{O}_{X}\right)
$$

where $\mathcal{F} \in \mathrm{D}_{\mathbb{R} \text {-c }}^{\mathrm{b}}\left(\mathbb{C}_{X}\right)$.

In $[\mathrm{KS} 2]$, the full functoriality of $\mathcal{O}^{t}$ is established. We will only need the following result:

Proposition 3.1.2. Let $f: X \rightarrow Y$ be a smooth map between complex manifolds. Then there is a canonical isomorphism in $\mathrm{D}^{\mathrm{b}}\left(\mathrm{I}\left(\beta\left(\mathcal{D}_{X}\right)\right)\right)$ :

$$
\operatorname{RJH} \operatorname{com}_{\beta \mathcal{D}_{X}}\left(\beta \mathcal{D}_{X \rightarrow Y}, \mathcal{O}_{X}^{t}\right) \simeq f^{-1} \mathcal{O}_{Y}^{t} .
$$

By microlocalization we get an object $\mu \mathcal{O}_{X}^{t} \in \mathrm{D}^{\mathrm{b}}\left(\mathrm{I}\left(\mathbb{C}_{T^{*} X}\right)\right)$. It is not known if this object is concentrated in a single degree or not. Our first objective is to prove that the microlocalization of the formula (3.1.1) holds, i.e. for every $\mathcal{F} \in \mathrm{D}_{\mathbb{R}-\mathrm{c}}^{\mathrm{b}}\left(\mathbb{C}_{X}\right)$ we have the formula

$$
\mathrm{R} \mathcal{H o m}\left(\mu \mathcal{F}, \mu \mathcal{O}^{t}\right) \simeq \operatorname{thhom}\left(\mathcal{F}, \mathcal{O}_{X}\right),
$$


where $\operatorname{t\mu hom}\left(\mathcal{F}, \mathcal{O}_{X}\right)$ is Andronikof's tempered version of the functor $\operatorname{\mu hom}\left(\mathcal{F}, \mathcal{O}_{X}\right)$ (see $\left.[\mathrm{An} 2]\right)$. The proof is similar to the proof of the formula

$$
\mathrm{RHom}(\mu \mathcal{F}, \mu \mathcal{G}) \simeq \mu h o m(\mathcal{F}, \mathcal{G})
$$

from $[\mathrm{K} 5]$ where $\mathcal{F}, \mathcal{G} \in \mathrm{D}^{\mathrm{b}}\left(\mathbb{C}_{X}\right)$. First recall the normal deformation of the diagonal in $X \times X$ that can be visualized by the following diagram

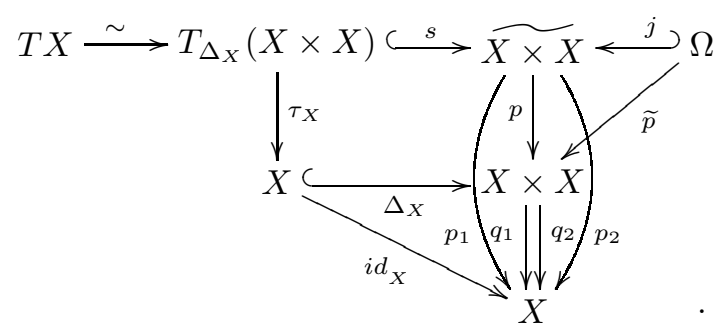

Recall that $\widetilde{X \times X}$ is equipped with a map $t: \widetilde{X \times X} \rightarrow \mathbb{R}$ and $\Omega$ is the open set $t^{-1}\left(\mathbb{R}_{>0}\right)$. Note that $\tilde{p}, p_{1}$ and $p_{2}$ are smooth but $p$ is not. Also, the square is not Cartesian. We use the same notations when we deform the diagonal in $T^{*} X \times T^{*} X$.

We will need the following lemma:

Lemma 3.1.3. Let $\mathcal{F} \in \mathrm{D}_{\mathbb{R}-c}^{\mathrm{b}}\left(\mathbb{C}_{T^{*} X}\right)$ and $\mathcal{G} \in \mathrm{D}^{\mathrm{b}}\left(I\left(\mathbb{C}_{T^{*} X}\right)\right)$. Then

$$
\operatorname{RH} \mathcal{H o m}(\mathcal{F}, \mu \mathcal{G}) \simeq \omega_{X}^{-1}\left(s^{-1} \operatorname{RH} \mathcal{H} o m\left(\left(p_{2}^{-1} \mathcal{F}\right)_{\Omega}, p^{-1} q_{1}^{!} \mathcal{G}\right)\right)^{\wedge} .
$$

$\operatorname{Here}(\cdot)^{\wedge}$ denotes the Fourier-Sato transform (see for instance [KS1]).

Proof. Set

$$
\begin{aligned}
P & =\left\{(x ; v) \in T T^{*} X \mid\langle v, \omega(x)\rangle \leqslant 0\right\}, \\
P^{\prime} & =\left\{(x ; v) \in T T^{*} X \mid\langle v, \omega(x)\rangle \geqslant 0\right\} .
\end{aligned}
$$

We have

$$
\begin{aligned}
& \mathrm{R} \mathcal{H} o m(\mathcal{F}, \mu \mathcal{G}) \simeq \mathrm{R} \mathcal{H} o m\left(\mathcal{F}, \mathrm{R} q_{1 ! !}\left(q_{2}^{-1} \mathcal{G} \otimes \mathrm{R} p_{! !}\left(\mathbb{C}_{\bar{\Omega}}\right) \otimes \beta\left(\mathbb{C}_{P} \otimes \mathrm{R} s_{*} \omega_{T T^{*} X \mid T^{*} X}\right)\right.\right. \\
& \simeq \mathrm{R} \mathcal{H} \text { om } \mathcal{F}, \mathrm{R} p_{1 ! !}\left(p_{2}^{-1} \mathcal{G} \otimes \mathbb{C}_{\bar{\Omega}} \otimes \beta\left(\mathbb{C}_{P} \otimes \mathrm{R} s_{*} \omega_{T T^{*} X \mid T^{*} X}\right)\right) \\
& \simeq \mathrm{RH} \mathcal{H} \text { om } \mathcal{F}, \mathrm{R} p_{2 ! !}\left(p_{1}^{-1} \mathcal{G} \otimes \mathbb{C}_{\bar{\Omega}} \otimes \beta\left(\mathbb{C}_{P^{\prime}} \otimes \mathrm{R} s_{*} \omega_{T T^{*} X \mid T^{*} X}\right)\right) \\
& \simeq \mathrm{R} p_{2 !} \mathrm{R} \mathcal{H} \text { om }\left(p_{2}^{-1} \mathcal{F}, p_{1}^{-1} \mathcal{G} \otimes \mathbb{C}_{\bar{\Omega}} \otimes \beta\left(\mathbb{C}_{P^{\prime}} \otimes \mathrm{R} s_{*} \omega_{T T^{*} X \mid T^{*} X}\right)\right) \\
& \left.\simeq \mathrm{R} p_{2 !}\left(\mathrm{R} \mathcal{H} o m\left(p_{2}^{-1} \mathcal{F}, p_{1}^{-1} \mathcal{G} \otimes \mathbb{C}_{\bar{\Omega}}\right) \otimes \mathbb{C}_{P^{\prime}} \otimes \mathrm{R} s_{*} \omega_{T T^{*} X \mid T^{*} X}\right)\right) \text {. }
\end{aligned}
$$


Now note that locally on $\left(T^{*} X \times T^{*} X\right)^{\sim}$ the set $\Omega$ is convex and

$$
\mathrm{SS}_{0}\left(p_{1}^{-1} \mathcal{G}\right) \cap T_{\Omega}^{*}\left(T^{*} X \times T^{*} X\right)^{\sim} \subset T_{\left(T^{*} X \times T^{*} X\right) \sim\left(T^{*} X \times T^{*} X\right)^{\sim} .} .
$$

Hence

$$
\mathbb{C}_{\bar{\Omega}} \otimes p_{1}^{-1} \mathcal{G} \simeq \operatorname{RH} \mathcal{H o m}\left(\mathbb{C}_{\Omega}, \mathbb{C}_{X}\right) \otimes p_{1}^{-1} \mathcal{G} \simeq \operatorname{RJH} \mathcal{H o m}\left(\mathbb{C}_{\Omega}, p_{1}^{-1} \mathcal{G}\right)
$$

Moreover note that

$$
\omega_{T T^{*} X \mid T^{*} X} \simeq \tau_{T^{*} X}^{-1} \omega_{\Delta \mid T^{*} X \times T^{*} X}^{\otimes-1} \simeq s^{-1} \tilde{p}^{-1} q_{1}^{!} \mathbb{C}_{T^{*} X} .
$$

Hence

$$
\begin{aligned}
\operatorname{RHom}(\mathcal{F}, \mu \mathcal{G}) & \left.\simeq \operatorname{R} p_{2 !}\left(\operatorname{RH} \mathcal{H o m}\left(p_{2}^{-1} \mathcal{F} \otimes \mathbb{C}_{\Omega}, p_{1}^{-1} \mathcal{G}\right) \otimes \mathbb{C}_{P^{\prime}} \otimes \mathrm{R} s_{*} \omega_{T T^{*} X \mid T^{*} X}\right)\right) \\
& \simeq \operatorname{R} p_{2 !} \operatorname{R} s_{!}\left(s^{-1} \operatorname{RH} \mathcal{H} o m\left(p_{2}^{-1} \mathcal{F} \otimes \mathbb{C}_{\Omega}, p^{-1} q_{1}^{!} \mathcal{G}\right) \otimes \mathbb{C}_{P^{\prime}}\right) \\
& \simeq \operatorname{R} \tau_{!}\left(s^{-1} \operatorname{RHom}\left(p_{2}^{-1} \mathcal{F} \otimes \mathbb{C}_{\Omega}, p^{-1} q_{1}^{!} \mathcal{G}\right) \otimes \mathbb{C}_{P^{\prime}}\right) \\
& \simeq \omega_{X}^{-1}\left(s^{-1} \operatorname{R\mathcal {H}om}\left(\left(p_{2}^{-1} \mathcal{F}\right)_{\Omega}, p^{-1} q_{1}^{!} \mathcal{G}\right)\right)^{\wedge} .
\end{aligned}
$$

This shows the lemma.

Proposition 3.1.4. Let $\mathcal{F} \in \mathrm{D}_{\mathbb{R}-c}^{\mathrm{b}}\left(\mathbb{C}_{X}\right)$. Then

$$
\operatorname{RHom}\left(\mu \mathcal{F}, \mu \mathcal{O}^{t}\right)=\operatorname{thhom}\left(\mathcal{F}, \mathcal{O}_{X}\right) .
$$

Proof. Apply Lemma 3.1.3 to $\mathcal{F} \in \mathrm{D}^{\mathrm{b}}\left(\mathbb{C}_{X}\right)$. Then a standard calculation shows that

$$
\operatorname{RHom}\left(\pi^{-1} \mathcal{F}, \mu \mathcal{G}\right)=\left(s^{-1} \operatorname{RHom}\left(\left(p_{2}^{-1} \mathcal{F}\right)_{\Omega}, p^{-1} q_{1}^{!} \mathcal{G}\right)\right)^{\wedge} .
$$

When we apply this result to $\mathcal{O}_{X}^{t}$ and $\mathcal{F} \in \mathrm{D}_{\mathbb{R}-\mathrm{c}}^{\mathrm{b}}\left(\mathbb{C}_{X}\right)$ we get

$$
\begin{aligned}
& \operatorname{RH} \mathcal{H o m}\left(\pi^{-1} \mathcal{F}, \mu \mathcal{O}^{t}\right) \simeq\left(s^{-1} \operatorname{RHom}\left(\left(p_{2}^{-1} \mathcal{F}\right)_{\Omega_{X}}, p^{-1} q_{1}^{!} \mathcal{O}^{t}\right)\right)^{\wedge} \\
& \simeq\left(s^{-1} \mathrm{R} \mathcal{H} \operatorname{Com}_{\mathcal{D}_{\widetilde{X \times X}}}\left(\mathcal{D}_{\widetilde{X \times X}} \stackrel{p_{1}}{\rightarrow}, \operatorname{TH} \mathcal{H} \text { om }\left(\left(p_{2}^{-1} \mathcal{F}\right)_{\Omega_{X}}, \mathcal{O}_{\widetilde{X \times X}}\right) \otimes s^{-1} p^{-1} q_{1}^{!} \mathbb{C}_{X}\right)^{\wedge}\right. \\
& \simeq\left(s^{-1} \mathcal{D}_{X \stackrel{p_{1}}{\longleftarrow} \widetilde{X \times X}} \underset{\mathcal{D}_{\widetilde{X \times X}}}{\otimes} \mathrm{TH} \mathcal{H} \operatorname{Hom}\left(\left(p_{2}^{-1} \mathcal{F}\right)_{\Omega}, \mathcal{O}_{\widetilde{X \times X}}\right)[1]\right)^{\wedge} \\
& \simeq \operatorname{t\mu hom}\left(\mathcal{F}, \mathcal{O}_{X}\right) \text {. }
\end{aligned}
$$

Following [KS2], we set for a locally free $\mathcal{O}_{X}$-module $\mathcal{L}$ :

$$
\mathcal{L}^{t}=\mathcal{O}_{X}^{t} \underset{\beta \mathcal{O}_{X}}{\stackrel{\mathrm{L}}{\otimes}} \beta \mathcal{L} .
$$


Lemma 3.1.5. $\quad$ Let $\mathcal{L}$ a locally free $\mathcal{O}_{X}$-module of finite rank. Then there are natural isomorphisms

$$
\begin{gathered}
\mu \mathcal{L} \stackrel{\sim}{\longrightarrow} \mu \mathcal{O}_{X} \underset{\pi^{-1} \beta \mathcal{O}_{X}}{\stackrel{\mathrm{L}}{\otimes}} \pi^{-1} \beta \mathcal{L}, \\
\mu \mathcal{L}^{t} \stackrel{\sim}{\longrightarrow} \mu \mathcal{O}_{X}^{t} \underset{\pi^{-1} \beta \mathcal{O}_{X}}{\stackrel{\mathrm{L}}{\otimes}} \pi^{-1} \beta \mathcal{L} .
\end{gathered}
$$

Proof. Let us show the second isomorphism, the proof of the first being similar since $\mathcal{L} \simeq \mathcal{O}_{X} \underset{\beta \mathcal{O}_{X}}{\stackrel{\mathrm{L}}{\otimes}} \beta \mathcal{L}$. By definition

$$
\mu\left(\mathcal{O}_{X}^{t} \underset{\beta \mathcal{O}_{X}}{\otimes} \beta \mathcal{L}\right) \simeq \mathrm{R} q_{1 ! !}\left(\mathrm{K}_{X} \otimes q_{2}^{-1} \pi^{-1}\left(\mathcal{O}_{X}^{t} \underset{\beta \mathcal{O}_{X}}{\otimes} \beta \mathcal{L}\right)\right) .
$$

But since $\mathrm{K}_{X} \simeq \mathrm{K}_{X} \otimes \beta \mathbb{C}_{\Delta}$ and

$$
\begin{aligned}
\beta \mathbb{C}_{\Delta} \otimes & \left(q_{2}^{-1} \pi^{-1} \mathcal{O}_{X}^{t} \underset{\beta q_{2}^{-1} \pi^{-1} \mathcal{O}_{X}}{\stackrel{\mathrm{L}}{\otimes}} \beta q_{2}^{-1} \pi^{-1} \mathcal{L}\right) \\
& \simeq\left(\left(\beta \mathbb{C}_{\Delta} \otimes q_{2}^{-1} \pi^{-1} \mathcal{O}_{X}^{t}\right) \underset{\beta q_{1}^{-1} \pi^{-1} \mathcal{O}_{X}}{\otimes} \beta q_{1}^{-1} \pi^{-1} \mathcal{L}\right)
\end{aligned}
$$

we get the second isomorphism.

\section{§3.2. $\varepsilon_{X}$-modules}

The ring $\mathcal{E}_{X}$ of microdifferential operators on $T^{*} X$ has been defined in [SKK]. For a short introduction to the theory of $\varepsilon_{X}$-modules we refer to [K3], for a more detailed study see also [Sch]. The ring $\mathcal{E}_{X}$ has many "good" properties, for instance it is coherent and Noetherian. For our purpose it is convenient to consider microdifferential operators outside the zero-section, hence when we write $\mathcal{E}_{X}$, we consider $\left.\mathcal{E}_{X}\right|_{T^{*} X}$.

We will also consider two variants of this ring. In the sequel we will identify $T^{*} X$ with $T_{\Delta_{X}}^{*}(X \times X)$ by the map

$$
\delta^{a}: T^{*} X \stackrel{\sim}{\longrightarrow} T_{\Delta_{X}}^{*}(X \times X) \hookrightarrow T^{*} X \times T^{*} X ; \quad(x ; \xi) \mapsto((x ; \xi),(x ;-\xi)) .
$$

The functor $\delta^{a-1}$ is often omitted for complexes with support on $T^{*} X$.

One defines the ring $\mathcal{E}_{X}^{\mathbb{R}}$ on $T^{*} X$ as

$$
\mathcal{E}_{X}^{\mathbb{R}} \simeq \mathrm{H}^{d_{X}} \mu h o m\left(\mathbb{C}_{\Delta_{X}}, \mathcal{O}_{X \times X}^{\left(d_{X}, 0\right)}\right) \simeq \mathrm{H}^{d_{X}} \mathrm{R} \mathcal{H} \operatorname{com}\left(\pi^{-1} \mathbb{C}_{\Delta_{X}}, \mu \mathcal{O}_{X \times X}^{\left(d_{X}, 0\right)}\right) .
$$


In [An2] Andronikof introduced the ring $\mathcal{E}_{X}^{\mathbb{R}, f}$ on $T^{*} X$ of tempered microdifferential operators as

$$
\mathcal{E}_{X}^{\mathbb{R}, f}=\mathrm{H}^{d_{X}}\left(\operatorname{t\mu hom}\left(\mathbb{C}_{\Delta}, \mathcal{O}_{X \times X}\right) \underset{\mathcal{O}_{X \times X}}{\stackrel{\mathrm{L}}{\otimes}} \mathcal{O}_{X \times X}^{\left(d_{X}, 0\right)}\right) .
$$

Proposition 3.2.1. We have

$$
\mathcal{E}_{X}^{\mathbb{R}, f} \simeq \mathrm{H}^{d_{X}} \mathrm{R} \mathcal{H} \operatorname{oom}\left(\pi^{-1} \mathbb{C}_{\Delta_{X}}, \mu \mathcal{O}_{X \times X}^{t,\left(d_{X}, 0\right)}\right) .
$$

Proof. Follows from Proposition 3.1.4 and Lemma 3.1.5.

Recall some basic properties (cf. [An2]):

(i) We have $\mathrm{R}^{i} \gamma_{*} \mathcal{E}_{X}^{\mathbb{R}, f} \simeq 0$ for $i \neq 0$ and $\mathcal{E}_{X} \simeq \gamma^{-1} \gamma_{*} \mathcal{E}_{X}^{\mathbb{R}, f}$.

(ii) The rings $\mathcal{E}_{X}^{\mathbb{R}}$ and $\mathcal{E}_{X}^{\mathbb{R}, f}$ are faithfully flat over $\mathcal{E}_{X}$.

(iii) The ring $\mathcal{E}_{X}$ (and therefore $\mathcal{E}_{X}^{\mathbb{R}}, \mathcal{E}_{X}^{\mathbb{R}, f}$ ) is a $\pi^{-1} \mathcal{D}_{X}$-module.

A priori, $\mathcal{E}_{X}$-modules are defined on the cotangent space $T^{*} X$. But since we have $\gamma^{-1} \gamma_{*} \mathcal{E}_{X} \simeq \mathcal{E}_{X}$, coherent $\mathcal{E}_{X}$-modules (hence in particular regular holonomic $\mathcal{E}_{X}$-modules) are conic objects, hence it is often convenient to work on the projective bundle $P^{*} X$ or on $\mathbb{C}^{\times}$-conic sets. We will always work outside the zero section.

Let $\mathcal{M}$ be a coherent $\mathcal{E}_{X}$-module. Then its support $\operatorname{supp}(\mathcal{M})$ is called its characteristic variety. If $\mathcal{M}$ is a coherent $\mathcal{D}_{X}$-module, then the characteristic variety of $\mathcal{E}_{X} \otimes_{\pi^{-1} \mathcal{D}_{X}} \pi^{-1} \mathcal{M}$ coincides with the characteristic variety of $\mathcal{M}$ as a $\mathcal{D}_{X}$-module. The main result about the characteristic variety of $\mathcal{E}_{X}$-modules is (see $[\mathrm{SKK}])$ :

Proposition 3.2.2. Let $\mathcal{M}$ be a coherent $\mathcal{E}_{X}$-module. Then its characteristic variety is a closed, analytic, involutive, $\mathbb{C}^{\times}$-conic subset of $T^{*} X$.

Definition 3.2.3. Let $\mathcal{M}$ be a coherent $\mathcal{E}_{X}$-module. One says that $\mathcal{M}$ is holonomic if its characteristic variety is Lagrangian.

Regular holonomic $\mathcal{E}_{X}$-modules ${ }^{7}$ (or "holonomic systems with regular singularities") have been studied in $[\mathrm{KK}]$. A $\mathcal{D}_{X}$-module is regular holonomic if and only if its associated $\mathcal{E}_{X}$-module is regular holonomic.

\footnotetext{
${ }^{7}$ A holonomoic $\mathcal{E}_{X}$-module $\mathcal{M}$ is regular holonomic if locally there exists a coherent $\mathcal{E}(0)$ module $\mathcal{L} \subset \mathcal{M}$ such that $\mathcal{M}=\mathcal{E}_{X} \mathcal{L}$ and for every homogenous holomorphic function $f \in \mathcal{O}_{\dot{T}^{*} X}(0)$ with $\left.f\right|_{\operatorname{supp}(\mathcal{M})}=0$ we have $f(\mathcal{L} / \mathcal{E}(-1) \mathcal{L})=0$.
} 
Theorem 3.2.4. Let $\mathcal{M}$ be a regular holonomic $\mathcal{E}_{X}$-module such that its characteristic variety is in generic position at a point $p \in \dot{T}^{*} X$. Then there exists a regular holonomic $\mathcal{D}_{X}$-module $\widetilde{\mathcal{M}}$ such that

$$
\mathcal{M} \simeq \mathcal{E}_{X} \underset{\pi^{-1} \mathcal{D}_{X}}{\otimes} \tilde{\mathcal{M}}
$$

Regular holonomic $\mathcal{E}_{X}$-modules form a stack of abelian categories. This stack is invariant by quantized contact transformations. Hence modulo a contact transformation a regular holonomic $\mathcal{E}_{X}$-module is locally isomorphic to

$$
\mathcal{E}_{X} \underset{\pi^{-1} \mathcal{D}_{X}}{\otimes} \operatorname{RH}(\mathcal{F})
$$

where $\mathcal{F}$ is a perverse sheaf. For our purpose this could be taken as a definition of a regular holonomic system. Finally let us recall the following theorem.

Proposition 3.2.5. ([An2], Théorème 4.2.6 and Proposition 5.6.1) Let $\mathcal{F} \in \mathrm{D}_{\mathbb{C}-c}^{\mathrm{b}}\left(\mathbb{C}_{X}\right)$. Then

$$
\operatorname{t\mu hom}\left(\mathcal{F}, \mathcal{O}_{X}\right) \simeq \mathcal{E}_{X}^{\mathbb{R}, f} \stackrel{\mathrm{L}}{\otimes} \underset{\pi^{-1} \mathcal{D}_{X}}{\operatorname{THom}}\left(\mathcal{F}, \mathcal{O}_{X}\right) .
$$

If $\mathcal{F}$ is perverse then thhom $\left(\mathcal{F}, \mathcal{O}_{X}\right)$ is concentrated in degree 0 and

$$
\mathrm{H}^{0} \operatorname{t\mu hom}\left(\mathcal{F}, \mathcal{O}_{X}\right) \simeq \mathcal{E}_{X}^{\mathbb{R}, f} \underset{\pi^{-1} \mathcal{D}_{X}}{\otimes} \pi^{-1} \mathrm{TH} \mathcal{H o m}\left(\mathcal{F}, \mathcal{O}_{X}\right) .
$$

§3.3. The microdifferential structure of $\mu \mathcal{O}_{X}$ and $\mu \mathcal{O}_{X}^{t}$

Recall that for any field $k$ an object $\mathcal{A} \in \mathrm{I}\left(k_{X}\right)$ is called a $k_{X}$-algebra if there exist morphisms

$$
k_{X} \rightarrow \mathcal{A} \quad \mathcal{A} \otimes \mathcal{A} \longrightarrow \mathcal{A}
$$

that satisfy the usual conditions of unit and associativity (for more details, see for instance [KS2], Section 5.4). For example, If $\mathcal{A}$ is a classical $k_{X^{-}}$-algebra in $\operatorname{Mod}\left(k_{X}\right)$ then $\beta \mathcal{A}$ is a $k_{X}$-algebra in $\mathrm{I}\left(k_{X}\right)$.

Let $\mathcal{A} \in \mathrm{I}\left(k_{X}\right)$ be a $k_{X}$-algebra. A left $\mathcal{A}$-module in $\mathrm{I}\left(k_{X}\right)$ is given by an object $\mathcal{M} \in \mathrm{I}\left(k_{X}\right)$ and a structure morphism

$$
\mathcal{A} \otimes \mathcal{M} \longrightarrow \mathcal{M}
$$

that satisfies the usual compatibility conditions with the structure morphisms of $\mathcal{A}$ (for more details, see loc. cit.). 
Similarly, one defines the notion of a $k_{X}$-algebra $\mathcal{A}$ in $\mathrm{D}^{\mathrm{b}}\left(\mathrm{I}\left(k_{X}\right)\right)$ and the notion of a left $\mathcal{A}$-module in $\mathrm{D}^{\mathrm{b}}\left(\mathrm{I}\left(k_{X}\right)\right)$. Note that if a $k_{X}$-algebra $\mathcal{A}$ in $\mathrm{D}^{\mathrm{b}}\left(\mathrm{I}\left(k_{X}\right)\right)$ is concentrated in a single degree, then it defines a $k_{X}$-algebra in $\mathrm{I}\left(k_{X}\right)$. However, even if $\mathcal{A}$ is concentrated in a single degree, an $\mathcal{A}$-module in $\mathrm{D}^{\mathrm{b}}\left(\mathrm{I}\left(k_{X}\right)\right)$ is in general not well-defined in the derived category of $\mathcal{A}$-modules in $\mathrm{I}\left(k_{X}\right)$. In order to avoid confusion, one often calls an $\mathcal{A}$-module in $\mathrm{D}^{\mathrm{b}}\left(\mathrm{I}\left(k_{X}\right)\right)$ a formal $\mathcal{A}$-module.

In this section we will show that $\mu \mathcal{O}_{X}$ is a $\beta \mathcal{E}_{X}^{\mathbb{R}}$-module in $\mathrm{I}\left(\mathbb{C}_{X}\right)$. The same strategy will show that $\mu \Theta_{X}^{t}$ is a formal $\beta \mathcal{E}_{X}^{\mathbb{R}, f}$-module in the derived category $\mathrm{D}^{\mathrm{b}}\left(\mathrm{I}\left(\mathbb{C}_{X}\right)\right)$. In particular its cohomology ind-sheaves are $\beta \mathcal{E}_{X}^{\mathbb{R}, f}$-modules.

We will need the tempered version of the integration morphism:

Proposition 3.3.1. There is a natural morphism in $\mathrm{D}^{\mathrm{b}}\left(\mathrm{I}\left(\mathbb{C}_{X}\right)\right)$

$$
\mathrm{R} f_{! !} \Omega_{X}^{t}\left[d_{X}\right] \longrightarrow \Omega_{Y}^{t}\left[d_{Y}\right]
$$

Proof. This is a simple version (not respecting $\mathcal{D}$-module structures) of the morphism established in [KS2]:

$$
\mathrm{R} f_{! !}\left(\Omega_{X}^{t} \underset{\beta \mathcal{D}_{X}}{\stackrel{L}{\otimes}} \beta \mathcal{D}_{X \rightarrow Y}\right)\left[d_{X}\right] \longrightarrow \Omega_{Y}^{t}\left[d_{Y}\right]
$$

Now consider complex manifolds $X, Y, Z$ of complex dimensions $d_{X}, d_{Y}, d_{Z}$ and the diagram

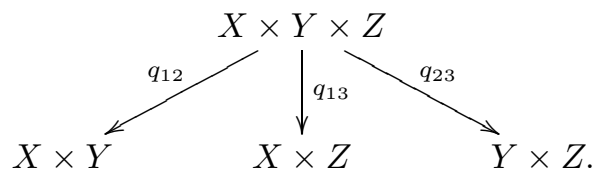

Lemma 3.3.2. $\quad$ The integration morphisms induce natural morphisms

$$
\begin{aligned}
& \mu \mathcal{O}_{X \times Y}^{\left(d_{X}, 0\right)} \stackrel{a}{\circ} \mu \mathcal{O}_{Y \times Z}^{\left(d_{Y}, 0\right)} \longrightarrow \mu \mathcal{O}_{X \times Z}^{\left(d_{X}, 0\right)}\left[-d_{Y}\right] \\
& \mu \mathcal{O}_{X \times Y}^{t,\left(d_{X}, 0\right)} \stackrel{a}{\circ} \mu \mathcal{O}_{Y \times Z}^{t,\left(d_{Y}, 0\right)} \longrightarrow \mu \mathcal{O}_{X \times Z}^{t,\left(d_{X}, 0\right)}\left[-d_{Y}\right]
\end{aligned}
$$

Proof. The two constructions being similar (and just an ind-variant of the construction used in Lemma 11.4.3. of [KS1]) we will only show how to define the second morphism.

First let us construct the natural morphism

$$
\mathcal{O}_{X \times Y}^{t,\left(d_{X}, 0\right)} \circ \mathcal{O}_{Y \times Z}^{t,\left(d_{Y}, 0\right)} \longrightarrow \mathcal{O}_{X \times Z}^{t,\left(d_{X}, 0\right)}\left[-d_{Y}\right]
$$


It can be obtained as follows

$$
\begin{aligned}
\mathcal{O}_{X \times Y}^{t,\left(d_{X}, 0\right)} \circ \mathcal{O}_{Y \times Z}^{t,\left(d_{Y}, 0\right)} & \simeq \mathrm{R} p_{13 ! !}\left(p_{12}^{-1} \mathcal{O}_{X \times Y}^{t,\left(d_{X}, 0\right)} \otimes p_{23}^{-1} \mathcal{O}_{Y \times Z}^{t,\left(d_{Y}, 0\right)}\right) \\
& \rightarrow \mathrm{R} p_{13 ! !} \mathcal{O}_{X \times Y \times Z}^{t,\left(d_{X}, d_{Y}, 0\right)} \\
& \rightarrow \mathcal{O}_{X \times Z}^{t,\left(d_{X}, 0\right)}\left[-d_{Y}\right]
\end{aligned}
$$

where the last morphism is the integration morphism of Proposition 3.3.1. Using the microlocal composition formula (Theorem A.2.5) we get the morphism

$$
\mu \mathcal{O}_{X \times Y}^{t,\left(d_{X}, 0\right)} \stackrel{a}{\circ} \mu \mathcal{O}_{Y \times Z}^{t,\left(d_{Y}, 0\right)} \longrightarrow \mu\left(\mathcal{O}_{X \times Y}^{t,\left(d_{X}, 0\right)} \circ \mathcal{O}_{Y \times Z}^{t,\left(d_{Y}, 0\right)}\right) \longrightarrow \mu \mathcal{O}_{X \times Z}^{t,\left(d_{X}, 0\right)}\left[-d_{Y}\right] .
$$

Proposition 3.3.3. Consider kernels $\mathcal{K}_{1} \in \mathrm{D}^{\mathrm{b}}\left(\mathrm{I}\left(\mathbb{C}_{T^{*} X \times T^{*} Y}\right)\right)$ and $\mathcal{K}_{2} \in \mathrm{D}^{\mathrm{b}}\left(\mathrm{I}\left(\mathbb{C}_{T^{*} Y \times T^{*} Z}\right)\right)$. There are natural morphisms

$$
\begin{aligned}
& \operatorname{RJH} \mathcal{H o m}\left(\mathcal{K}_{1}, \mu \mathcal{O}_{X \times Y}^{\left(d_{X}, 0\right)}\right) \stackrel{a}{\circ} \operatorname{RJH} \mathcal{H} o m\left(\mathcal{K}_{2}, \mu \mathcal{O}_{Y \times Z}^{\left(d_{Y}, 0\right)}\right) \\
& \longrightarrow \mathrm{RJH} \mathcal{H o m}\left(\mathcal{K}_{1} \stackrel{a}{\circ} \mathcal{K}_{2}, \mu \mathcal{O}_{X \times Z}^{\left(d_{X}, 0\right)}\right)\left[-d_{Y}\right] \\
& \operatorname{RJ\mathcal {H}} \operatorname{om}\left(\mathcal{K}_{1}, \mu \Theta_{X \times Y}^{t,\left(d_{X}, 0\right)}\right) \stackrel{a}{\circ} \operatorname{RJH} \mathcal{H} o m\left(\mathcal{K}_{2}, \mu \Theta_{Y \times Z}^{t,\left(d_{Y}, 0\right)}\right) \\
& \longrightarrow \operatorname{RJ} \mathcal{H} \text { om }\left(\mathcal{K}_{1}{ }^{a} \mathcal{K}_{2}, \mu \mathcal{O}_{X \times Z}^{t,\left(d_{X}, 0\right)}\right)\left[-d_{Y}\right]
\end{aligned}
$$

These morphisms satisfy the obvious associativity condition (analogous to Lemma 11.4 .3 in [KS1]).

Proof. Note that there is a natural morphism

$$
\operatorname{RJH} \mathcal{H o m}\left(\mathcal{F}_{1}, \mathcal{G}_{1}\right) \stackrel{a}{\circ} \operatorname{RJH} \mathcal{H o m}\left(\mathcal{F}_{2}, \mathcal{G}_{2}\right) \longrightarrow \operatorname{RJH} \mathcal{H o m}\left(\mathcal{F}_{1} \stackrel{a}{\circ} \mathcal{F}_{2}, \mathcal{G}_{1} \stackrel{a}{\circ} \mathcal{G}_{2}\right) .
$$

Combining this morphism with the morphisms of Lemma 3.3.2, we get the desired arrows.

The associativity condition is tedious to write down. It is a straightforward consequence of the corresponding associativity conditions of the morphisms involved in the construction.

Remark 3.3.4. Consider $\mathcal{K}_{1} \in \mathrm{D}^{\mathrm{b}}\left(\mathbb{C}_{X \times Y}\right)$ and $\mathcal{K}_{2} \in \mathrm{D}^{\mathrm{b}}\left(\mathbb{C}_{Y \times Z}\right)$. Applying the functor $\alpha$ to the first morphism of Proposition 3.3.3 (and replacing $\mathcal{K}_{i}$ by $\left.\mu \mathcal{K}_{i}, i=1,2\right)$, we get a morphism

$$
\begin{aligned}
& \operatorname{R} p_{13 !}\left(p_{12}^{a-1} \mu h o m\left(\mathcal{K}_{1}, \mathcal{O}_{X \times Y}^{\left(d_{X}, 0\right)}\right) \otimes p_{23}^{-1} \mu h o m\left(\mathcal{K}_{2}, \mathcal{O}_{Y \times Z}^{\left(d_{Y}, 0\right)}\right)\right) \\
& \longrightarrow \mu h o m\left(\mathcal{K}_{1} \circ \mathcal{K}_{2}, \mathcal{O}_{X \times Z}^{\left(d_{X}, 0\right)}\right)\left[-d_{Y}\right] .
\end{aligned}
$$

This morphism is naturally isomorphic to the morphism of [KS1], Lemma 11.4.3. 
In the situation of Proposition 3.3.3 consider $Z=\{p t\}$ and $\mathcal{K}_{2}=\mu \mathcal{O}_{Y}$ (resp. $\mathcal{K}_{2}=\mu \mathcal{O}_{Y}^{t}$ ). Applying $\alpha$ we get natural morphisms

$$
\begin{aligned}
& \mathrm{R} p_{1 !}\left(\mathrm{R} \mathcal{H} \operatorname{com}\left(\mathcal{K}, \mu \mathcal{O}_{X \times Y}^{\left(0, d_{Y}\right)}\right) \otimes p_{2}^{a-1} \mathrm{R} \mathcal{H} \operatorname{com}\left(\mu \mathcal{O}_{Y}, \mu \mathcal{O}_{Y}\right)\right) \\
& \longrightarrow \mathrm{R} \mathcal{H} o m\left(\mathcal{K}\left[d_{Y}\right]^{a} \stackrel{\mu}{\mu} \mathcal{O}_{Y}, \mu \mathcal{O}_{X}\right), \\
& \mathrm{R} p_{1 !}\left(\operatorname{RH} \mathcal{H} o m\left(\mathcal{K}, \mu \Theta_{X \times Y}^{t,\left(0, d_{Y}\right)}\right) \otimes p_{2}^{a-1} \operatorname{RH} \mathcal{H} o m\left(\mu \Theta_{Y}^{t}, \mu \Theta_{Y}^{t}\right)\right) \\
& \longrightarrow \mathrm{R} \mathcal{H} \circ \mathrm{Km}\left(\mathcal{K}\left[d_{Y}\right]^{\circ} \stackrel{\circ}{\mu} \mathcal{O}_{Y}^{t}, \mu \mathcal{O}_{X}^{t}\right) \text {. }
\end{aligned}
$$

Taking $X=Y$ and $\mathcal{K}=\pi^{-1} \mathbb{C}_{\Delta_{X}}$ we get the morphisms

$$
\begin{aligned}
& \mathcal{E}_{X}^{\mathbb{R}} \otimes \mathrm{R} \mathcal{H} \text { om }\left(\mu \mathcal{O}_{X}, \mu \mathcal{O}_{X}\right) \longrightarrow \mathrm{R} \mathcal{H} o m\left(\mu \mathcal{O}_{X}, \mu \mathcal{O}_{X}\right), \\
& \mathcal{E}_{X}^{\mathbb{R}, f} \otimes \mathrm{R} \mathcal{H} o m\left(\mu \mathcal{O}_{X}^{t}, \mu \mathcal{O}_{X}^{t}\right) \longrightarrow \mathrm{R} \mathcal{H} o m\left(\mu \mathcal{O}_{X}^{t}, \mu \mathcal{O}_{X}^{t}\right) .
\end{aligned}
$$

Note that for any $\mathcal{F} \in \mathrm{D}^{\mathrm{b}}\left(\mathrm{I}\left(\mathbb{C}_{X}\right)\right)$ the identity of $\mathcal{F}$ defines a natural morphism $\mathbb{C}_{\Delta_{X}} \rightarrow \mathrm{R} \mathcal{H o m}(\mathcal{F}, \mathcal{F})$. Hence we get the structure morphisms

$$
\begin{gathered}
\beta \mathcal{E}_{X}^{\mathbb{R}} \otimes \mu \mathcal{O}_{X} \longrightarrow \mu \mathcal{O}_{X}, \\
\beta \mathcal{E}_{X}^{\mathbb{R}, f} \otimes \mu \mathcal{O}_{X}^{t} \longrightarrow \mu \mathcal{O}_{X}^{t} .
\end{gathered}
$$

In order to prove that the two morphisms (3.3.1) and (3.3.2) define structures of formal modules in $\mathrm{D}^{\mathrm{b}}\left(\mathrm{I}\left(\mathbb{C}_{X}\right)\right)$ one uses the associativity of the construction in Proposition (3.3.3). The proof that the action is unitary is the same as for t $\mu$ hom in [An2] (Proposition 4.2.4) and $\mu$ hom in [KS1] (Proposition 11.4.4) and goes back to $[\mathrm{SKK}]$. Therefore we get:

Proposition 3.3.5. The object $\mu \mathcal{O}_{X}$ (resp. $\left.\mu \Omega_{X}\right)$ is a formal left (resp. right) $\beta \mathcal{E}_{X}^{\mathbb{R}}$-module in $\mathrm{D}^{\mathrm{b}}\left(\mathrm{I}\left(\mathbb{C}_{X}\right)\right.$ ) and $\mu \mathcal{O}_{X}^{t}$ (resp. $\mu \Omega_{X}^{t}$ ) is a formal left (resp. right) $\beta \mathcal{E}_{X}^{\mathbb{R}, f}$-module in $\mathrm{D}^{\mathrm{b}}\left(\mathrm{I}\left(\mathbb{C}_{X}\right)\right)$.

Corollary 3.3.6. Let $\mathcal{F} \in \mathrm{D}^{\mathrm{b}}\left(\mathrm{I}\left(\mathbb{C}_{T^{*} X}\right)\right)$. Then

(i) the complex $\operatorname{RJH} \mathcal{H o m}\left(\mathcal{F}, \mu \mathcal{O}_{X}\right)$ (resp. $\operatorname{RJH} \operatorname{Hom}\left(\mathcal{F}, \mu \mathcal{O}_{X}^{t}\right)$ ) is a formal left $\beta \mathcal{E}_{X}^{\mathbb{R}}$-module (resp. $\beta \mathcal{E}_{X}^{\mathbb{R}, f}$-module) in $\mathrm{D}^{\mathrm{b}}\left(\mathrm{I}\left(\mathbb{C}_{X}\right)\right)$,

(ii) the complex $\mathrm{RJH} \mathcal{H} o m\left(\mathcal{F}, \mu \Omega_{X}\right)$ (resp. $\left.\operatorname{RJH} \mathcal{H o m}\left(\mathcal{F}, \mu \Omega_{X}^{t}\right)\right)$ is a formal right $\beta \mathcal{E}_{X}^{\mathbb{R}}$-module (resp. $\beta \mathcal{E}_{X}^{\mathbb{R}, f}$-module) in $\mathrm{D}^{\mathrm{b}}\left(\mathrm{I}\left(\mathbb{C}_{X}\right)\right)$. 
(iii) The object $\mu \mathcal{O}_{X}$ (resp. $\left.\mu \Omega_{X}\right)$ is a left (resp. right) $\beta \mathcal{E}_{X}^{\mathbb{R}}$-module in $\mathrm{I}\left(\mathbb{C}_{T^{*} X}\right)$ and for every $\mathcal{F} \in \mathrm{D}^{\mathrm{b}}\left(\mathbb{C}_{X}\right)$ the complex $\mu$ hom $\left(\mathcal{F}, \mathcal{O}_{X}\right)$ is well defined in the derived category of $\mathcal{E}_{X}^{\mathbb{R}}$-modules.

(iv) Let $\mathcal{F} \in \mathrm{D}^{\mathrm{b}}\left(\mathrm{I}\left(\mathbb{C}_{T^{*} X}\right)\right)$ such that $\mathrm{R} \mathcal{H} \operatorname{om}\left(\mathcal{F}, \mu \mathcal{O}_{X}^{t}\right)$ is concentrated in a single degree (for instance if $\mathcal{F}$ is a microlocal perverse sheaf (see Lemma 3.6.1 below)). Then the natural morphism

$$
\mathrm{R} \mathcal{H} o m\left(\mathcal{F}, \mu \mathcal{O}_{X}^{t}\right) \longrightarrow \mathrm{R} \mathcal{H} o m\left(\mathcal{F}, \mu \mathcal{O}_{X}\right)
$$

is well defined in the derived category of $\mathcal{E}_{X}$-modules.

We also get

Proposition 3.3.7. The natural morphism

$$
\begin{aligned}
& \operatorname{R} p_{13 ! !}\left(p_{12}^{a-1} \operatorname{RJ\mathcal {H}om}\left(\mathcal{K}_{1}, \mu \mathcal{O}_{X \times Y}^{\left(d_{X}, 0\right)}\right) \otimes p_{23}^{-1} \operatorname{RJH} \mathcal{H} o m\left(\mathcal{K}_{2}, \mu \mathcal{O}_{Y \times Z}^{\left(d_{Y}, 0\right)}\right)\right) \\
& \longrightarrow \operatorname{RJ} \mathcal{H} \operatorname{om}\left(\mathcal{K}_{1} \stackrel{a}{\circ} \mathcal{K}_{2}, \mu \mathcal{O}_{X \times Z}^{\left(d_{X}, 0\right)}\right)\left[-d_{Y}\right]
\end{aligned}
$$

factors through

$$
\mathrm{R} p_{13 ! !}\left(p_{12}^{a-1} \operatorname{RJH} \mathcal{H} o m\left(\mathcal{K}_{1}, \mu \mathcal{O}_{X \times Y}^{\left(d_{X}, 0\right)}\right) \underset{p_{2}^{-1} \beta \mathcal{E}_{X}^{\mathbb{R}}}{\stackrel{\mathrm{L}}{\otimes}} p_{23}^{-1} \operatorname{RJH} \mathcal{H} o m\left(\mathcal{K}_{2}, \mu \mathcal{O}_{Y \times Z}^{\left(d_{Y}, 0\right)}\right)\right) .
$$

Proof. This is a consequence of the associativity condition of Proposition 3.3.3.

§3.4. Quantized contact transformations for $\mu \mathcal{O}_{X}$ and $\mu \mathcal{O}_{X}^{t}$

In this section we will adapt Section 11.4 of [KS1] to study the behaviour of $\mu \mathcal{O}_{X}$ and $\mu \mathcal{O}_{X}^{t}$ under complex contact transformations. We will restrict ourselves now to $\mu \mathcal{O}_{X}$, the study of $\mu \mathcal{O}_{X}^{t}$ being similar.

Hence to any contact transformation $\chi: \Omega_{Y} \stackrel{\sim}{\longrightarrow} \Omega_{X}$ we want to attach a kernel $\mathcal{K}$ and an isomorphism

$$
\mu \mathcal{K}^{a} \sim \mu \mathcal{O}_{Y} \stackrel{\sim}{\longrightarrow} \mu \mathcal{O}_{X}
$$

that is compatible with the action of $\beta \mathcal{E}_{X}\left(\right.$ resp. $\left.\beta \mathcal{E}_{Y}\right)$ on $\mu \mathcal{O}_{X}\left(\right.$ resp. $\left.\mu \mathcal{O}_{Y}\right)$. Such a morphism has been constructed in $\mathrm{D}^{\mathrm{b}}\left(\mathbb{C}_{X}, p\right)$ in Section 11.4 of [KS1].

Recall the morphism

$$
\begin{aligned}
\mathrm{R} p_{1 !}\left(\mathrm{R} \mathcal{H} o m\left(\mu \mathcal{K}, \mu \mathcal{O}_{X \times Y}^{\left(0, d_{Y}\right)}\right) \otimes p_{2}^{a-1}\right. & \left.\mathrm{R} \mathcal{H} \operatorname{com}\left(\mu \mathcal{O}_{Y}, \mu \mathcal{O}_{Y}\right)\right) \\
& \longrightarrow \mathrm{R} \mathcal{H} \operatorname{com}\left(\mu \mathcal{K}\left[d_{Y}\right]_{\circ}^{a} \mu \mathcal{O}_{Y}, \mu \mathcal{O}_{X}\right)
\end{aligned}
$$


Now suppose that $\mathrm{R} \mathcal{H} o m\left(\mathcal{K}, \mu \mathcal{O}_{X \times Y}^{\left(0, d_{Y}\right)}\right)$ is concentrated in positive degrees and that

$$
\operatorname{supp} \operatorname{RHom}\left(\mathcal{K}, \mu \mathcal{O}_{X \times Y}^{\left(0, d_{Y}\right)}\right) \rightarrow X
$$

is proper. Then by taking the 0-cohomology we get a morphism

$$
\begin{aligned}
p_{1 *}\left(\mathrm{H}^{0} \mathrm{R} \mathcal{H} \text { om }\left(\mu \mathcal{K}, \mu \mathcal{O}_{X \times Y}^{\left(0, d_{Y}\right)}\right) \otimes\right. & \left.p_{2}^{a-1} \mathrm{H}^{0} \mathrm{R} \mathcal{H} \operatorname{com}\left(\mu \mathcal{O}_{Y}, \mu \mathcal{O}_{Y}\right)\right) \\
& \longrightarrow \mathrm{H}^{0} \mathrm{R} \mathcal{H} \operatorname{Com}\left(\mu \mathcal{K}\left[d_{Y}\right]^{a}{ }^{\circ} \mu \mathcal{O}_{Y}, \mu \mathcal{O}_{X}\right) .
\end{aligned}
$$

Hence the identity of $\mu \mathcal{O}_{Y}$ and any section

$$
s \in \mathrm{H}^{0} \mathrm{R} \mathcal{H} \text { om }\left(\mu \mathcal{K}, \mu \mathcal{O}_{X \times Y}^{\left(0, d_{Y}\right)}\right) .
$$

define a morphism

$$
\varphi(s): \mathcal{K}\left[d_{Y}\right]^{\circ} \stackrel{a}{\circ} \mathcal{O}_{Y} \longrightarrow \mu \mathcal{O}_{X}
$$

Recall the morphism

$$
\begin{aligned}
p_{13 *}^{a}\left(p_{12}^{a-1} \mathrm{R} \mathcal{H} \operatorname{com}\right. & \left.\left(\mu \mathcal{K}_{1}, \mu \mathcal{O}_{X \times Y}^{\left(0, d_{Y}\right)}\right) \underset{\beta \varepsilon_{Y}^{\mathbb{R}}}{\otimes} p_{23}^{a-1} \operatorname{R\mathcal {H}om}\left(\mu \mathcal{K}_{2}, \mu \mathcal{O}_{Y \times Z}^{\left(0, d_{Z}\right)}\right)\right) \\
& \longrightarrow \operatorname{RJ\mathcal {H}om}\left(\mu \mathcal{K}_{1} \stackrel{a}{\circ} \mu \mathcal{K}_{2}\left[d_{Y}\right], \mu \mathcal{O}_{X \times Z}^{\left(0, d_{Z}\right)}\right) \\
& \longrightarrow \operatorname{RJ\mathcal {H}om}\left(\mu\left(\mathcal{K}_{1} \circ \mathcal{K}_{2}\right)\left[d_{Y}\right], \mu \mathcal{O}_{X \times Z}^{\left(0, d_{Z}\right)}\right) .
\end{aligned}
$$

Denote by $s \circ s^{\prime}$ the image of $s \otimes s^{\prime}$ by this morphism. Then we get

Proposition 3.4.1.

$$
\varphi\left(s \circ s^{\prime}\right)=\varphi(s) \circ\left(\mathcal{K}[n] \stackrel{a}{\circ} \varphi\left(s^{\prime}\right)\right)
$$

Proof. This follows from the fact that the morphism of Proposition 3.3.3 satisfies to the obvious associativity condition.

In the sequel we will only consider kernels $\mathcal{K} \in \mathrm{D}^{\mathrm{b}}\left(\mathbb{C}_{Y \times X}\right)$ satisfying

(i) $\mathcal{K}$ is $\mathbb{R}$-constructible,

(ii) $\left(\Omega_{Y} \times T^{*} X \cup T^{*} Y \times \Omega_{X}^{a}\right) \cap \mathrm{SS}(\mathcal{K}) \subset \Lambda$,

(iii) $\mathcal{K}$ is simple ${ }^{8}$ with shift 0 along $\Lambda$.

Note that given a $\mathbb{C}^{\times}$-conic Lagrangian subvariety $\Lambda$ that is associated to a contact transformation, for each $p \in \Omega_{X}$ there exist a $\mathbb{C}^{\times}$-conic open neighborhood $\Omega_{X}^{\prime}$ of $\mathbb{C}^{\times} p$ and a kernel $\mathcal{K}$ such that (i), (ii) and (iii) are satisfied.

\footnotetext{
${ }^{8}$ For the definition of simple sheaves see [KS1], Section 7.5.
} 
Theorem 3.4.2. $\quad$ For every $p \in \Omega_{Y}$ there exists a $\mathbb{C}^{\times}$-conic open neighborhood $\Omega_{Y}^{\prime} \subset \Omega_{Y}$ of $\mathbb{C}^{\times} p$ such that if we set $\Omega_{X}^{\prime}=\chi\left(\Omega_{X}\right)$ we can find sections $\left.s \in \mathrm{H}^{0} \mathrm{R} \mathcal{H} \operatorname{om}\left(\mu \mathcal{K}, \mu \mathcal{O}_{X \times Y}^{\left(d_{X}, 0\right)}\right)\right|_{\Omega_{X}^{\prime} \times \Omega_{Y}^{\prime}} \quad s^{t} \in \mathrm{H}^{0} \mathrm{R} \mathcal{H}$ om $\left.\left(\mu \mathcal{K}, \mu \mathcal{O}_{X \times Y}^{t,\left(d_{X}, 0\right)}\right)\right|_{\Omega_{X}^{\prime} \times \Omega_{Y}^{\prime}}$ such that the morphisms

$$
\begin{gathered}
\left.\left.\mathcal{E}_{X}^{\mathbb{R}}\right|_{\Omega_{X}} \longrightarrow p_{1 *} \mathrm{H}^{0} \mathrm{R} \mathcal{H} \operatorname{com}\left(\mu \mathcal{K}, \mu \mathcal{O}_{X \times Y}^{\left(d_{X}, 0\right)}\right)\right|_{\Omega_{X}^{\prime} \times \Omega_{Y}^{\prime}} ; \quad P \mapsto P s \\
\left.\left.\mathcal{E}_{X}^{\mathbb{R}, f}\right|_{\Omega_{X}} \longrightarrow p_{1 *} \mathrm{H}^{0} \mathrm{R} \mathcal{H} \operatorname{com}\left(\mu \mathcal{K}, \mu \mathcal{O}_{X \times Y}^{t,\left(d_{X}, 0\right)}\right)\right|_{\Omega_{X}^{\prime} \times \Omega_{Y}^{\prime}} ; \quad P \mapsto P s^{t} \\
\left.\left.\mathcal{E}_{Y}^{\mathbb{R}}\right|_{\Omega_{Y}} \longrightarrow p_{2 *} \mathrm{H}^{0} \mathrm{R} \mathcal{H} \operatorname{com}\left(\mu \mathcal{K}, \mu \mathcal{O}_{X \times Y}^{\left(d_{X}, 0\right)}\right)\right|_{\Omega_{X}^{\prime} \times \Omega_{Y}^{\prime}} ; \quad Q \mapsto s Q \\
\left.\left.\mathcal{E}_{Y}^{\mathbb{R}, f}\right|_{\Omega_{Y}} \longrightarrow p_{2 *} \mathrm{H}^{0} \mathrm{R} \mathcal{H} \operatorname{com}\left(\mu \mathcal{K}, \mu \mathcal{O}_{X \times Y}^{t,\left(d_{X}, 0\right)}\right)\right|_{\Omega_{X}^{\prime} \times \Omega_{Y}^{\prime}} ; \quad Q \mapsto s^{t} Q
\end{gathered}
$$

are isomorphisms and we get antiisomorphisms

$$
\begin{gathered}
\left.\left.\chi_{*} \mathcal{E}_{Y}^{\mathbb{R}}\right|_{\Omega_{Y}} \stackrel{\sim}{\longrightarrow} \mathcal{E}_{X}^{\mathbb{R}}\right|_{\Omega_{X}} ; \quad P \mapsto Q \text { such that } P s=s Q \\
\left.\left.\chi_{*} \mathcal{E}_{Y}^{\mathbb{R}, f}\right|_{\Omega_{Y}} \stackrel{\sim}{\longrightarrow} \mathcal{E}_{X}^{\mathbb{R}, f}\right|_{\Omega_{X}} ; \quad P \mapsto Q \text { such that } P s^{t}=s^{t} Q
\end{gathered}
$$

For such sections $s, s^{t}$ the morphism

$$
\varphi(s): \mu \mathcal{K}\left[d_{Y}\right]^{a} \mu \mathcal{O}_{Y} \longrightarrow \mu \mathcal{O}_{X} \quad \varphi\left(s^{t}\right): \mu \mathcal{K}\left[d_{Y}\right] \stackrel{a}{\circ} \mu \mathcal{O}_{Y}^{t} \longrightarrow \mu \mathcal{O}_{X}^{t}
$$

is an isomorphism of $\beta \mathcal{E}_{X}^{\mathbb{R}}$-modules (resp. $\beta \mathcal{E}_{X}^{\mathbb{R}, f}$-modules).

Corollary 3.4.3. Let $s, s^{t}$ be a section as in Theorem 3.4.2.

(i) Let $\mathcal{F}$ be a perverse sheaf. Then $\varphi(s)$ defines an isomorphism of $\mathcal{E}_{X}^{\mathbb{R}}$-modules

$$
\chi_{*} \mathrm{R} \mathcal{H} o m\left(\pi^{-1} \mu \mathcal{F}, \mu \mathcal{O}_{Y}\right) \simeq \mathrm{R} \mathcal{H} \text { om }\left(\mu \mathcal{K} \stackrel{a}{\circ} \mu \mathcal{F}, \mu \mathcal{O}_{X}\right)
$$

and $\varphi\left(s^{t}\right)$ defines an isomorphism of $\mathcal{E}_{X}^{\mathbb{R}, f}$

$$
\chi_{*} \operatorname{RHom}\left(\pi^{-1} \mu \mathcal{F}, \mu \mathcal{O}_{Y}^{t}\right) \simeq \operatorname{R\mathcal {H}om}\left(\mu \mathcal{K}_{\circ}^{a} \mu \mathcal{F}, \mu \mathcal{O}_{X}^{t}\right) .
$$

(ii) Let $\mathcal{M}$ be a coherent $\mathcal{E}_{Y}$-module. Then $\varphi(s)$ defines an isomorphism

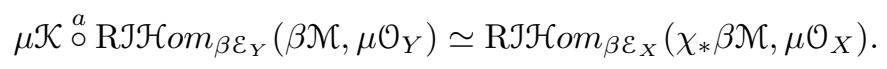

\section{§3.5. Classical Riemann-Hilbert Theorem and Ind-sheaves}

Using the functor $\mu$ we can reformulate the isomorphisms of the classical Riemann-Hilbert Theorem from the microlocal point of view. 


\section{Lemma 3.5.1.}

(1) Let $\mathcal{F} \in \mathrm{D}_{\mathbb{C}-c}^{\mathrm{b}}\left(\mathbb{C}_{X}\right)$. Then

$$
\begin{aligned}
& \mathrm{R} \mathcal{H} o m\left(\mu \mathcal{F}, \mu \mathcal{O}_{X}^{t}\right) \simeq \mathcal{E}_{X}^{\mathbb{R}, f} \underset{\pi^{-1} \mathcal{D}_{X}}{\stackrel{\mathrm{L}}{\otimes}} \pi^{-1} \mathrm{RH}(\mathcal{F}), \\
& \gamma^{-1} \mathrm{R} \gamma_{*} \mathrm{R} \mathcal{H} \text { om }\left(\mu \mathcal{F}, \mu \mathcal{O}^{t}\right) \simeq \mathcal{E}_{X} \underset{\pi^{-1} \mathcal{D}_{X}}{\stackrel{\mathrm{L}}{\otimes}} \pi^{-1} \mathrm{RH}(\mathcal{F}) .
\end{aligned}
$$

(2) Let $\mathcal{M}$ be a coherent $\mathcal{D}_{X}$-module. Then

$$
\begin{aligned}
& \operatorname{RJH}_{\mathcal{H}} m_{\beta \mathcal{E}_{X}^{\mathbb{R}, f}}\left(\beta \mathcal{E}_{X}^{\mathbb{R}, f} \underset{\pi^{-1} \beta \mathcal{D}_{X}}{\stackrel{\mathrm{L}}{\otimes}} \beta\left(\pi^{-1} \mathcal{N}\right), \mu \mathcal{O}_{X}\right) \simeq \mu(\operatorname{Sol}(\mathcal{M})), \\
& \operatorname{RJF}_{\mathcal{H}} \operatorname{mom}_{\beta \mathcal{E}_{X}}\left(\beta \mathcal{E}_{X} \underset{\pi^{-1} \beta \mathcal{D}_{X}}{\stackrel{\mathrm{L}}{\otimes}} \beta\left(\pi^{-1} \mathcal{M}\right), \mu \mathcal{O}_{X}\right) \simeq \mu(\operatorname{Sol}(\mathcal{M})) .
\end{aligned}
$$

Proof. (1) is a reformulation of Theorem 4.2.6 of [An2] in terms of indsheaves using the fact that $\gamma^{-1} \mathrm{R} \gamma_{*} \mathcal{E}_{X}^{\mathbb{R}, f} \simeq \mathcal{E}_{X}$ and (2) follows directly from the fact that $\mathcal{M}$ is a coherent $\mathcal{D}_{X}$-module.

Therefore we can now formulate Riemann-Hilbert Theorem in terms of ind-sheaves:

\section{Proposition 3.5.2.}

(1) Let $\mathcal{F}$ be a perverse sheaf on $X$. Then

$$
\begin{aligned}
& \operatorname{RJH} \operatorname{com}_{\beta \varepsilon_{X}^{\mathbb{R}, f}}\left(\beta \operatorname{RHom}\left(\mu \mathcal{F}, \mu \mathcal{O}_{X}^{t}\right), \mu \mathcal{O}_{X}\right) \simeq \mu \mathcal{F}, \\
& \operatorname{RJH} \operatorname{Hom}_{\beta \mathcal{E}_{X}}\left(\beta \gamma^{-1} \mathrm{R} \gamma_{*} \operatorname{R\mathcal {H}om}\left(\mu \mathcal{F}, \mu \mathcal{O}_{X}^{t}\right), \mu \mathcal{O}_{X}\right) \simeq \mu \mathcal{F} \text {. }
\end{aligned}
$$

(2) Let $\mathcal{M}$ be a regular holonomic $\mathcal{D}_{X}$-module. Then

$$
\begin{aligned}
& \mathrm{R} \mathcal{H} \text { om }\left(\mathrm{RJ \mathcal {H }} \operatorname{Com}_{\beta \mathcal{E}_{X}^{\mathbb{R}, f}}\left(\beta\left(\mathcal{E}_{X}^{\mathbb{R}, f} \underset{\pi^{-1} \mathcal{D}_{X}}{\mathrm{~L}} \pi^{-1} \mathcal{N}\right), \mu \mathcal{O}_{X}\right), \mu \mathcal{O}_{X}^{t}\right) \\
& \simeq \mathcal{E}_{X}^{\mathbb{R}, f} \underset{\pi^{-1} \mathcal{D}_{X}}{\stackrel{\mathrm{L}}{\otimes}} \pi^{-1} \mathcal{M}, \\
& \gamma^{-1} \mathrm{R} \gamma_{*} \mathrm{R} \mathcal{H} \text { om }\left(\mathrm{RJH} \operatorname{Hom}_{\beta \mathcal{E}_{X}}\left(\beta\left(\mathcal{E}_{X} \underset{\pi^{-1} \mathcal{D}_{X}}{\stackrel{\mathrm{L}}{\otimes}} \pi^{-1} \mathcal{M}\right), \mu \mathcal{O}_{X}\right), \mu \mathcal{O}_{X}^{t}\right) \\
& \simeq \mathcal{E}_{X} \underset{\pi^{-1} \mathcal{D}_{X}}{\stackrel{\mathrm{L}}{\otimes}} \pi^{-1} \mathcal{M} \text {. }
\end{aligned}
$$


Proof. The proof follows from Lemma 3.5.1.

Now let us formulate the comparison theorem for regular holonomic $\mathcal{E}_{X^{-}}$ modules in terms of ind-sheaves. The classical version ([An1], Proposition 5.6.3) states

Proposition 3.5.3. Let $\mathcal{M}$ be a regular holonomic $\mathcal{E}_{X}$-module and $\mathcal{G} \in$ $\mathrm{D}_{\mathbb{R}-c}^{\mathrm{b}}\left(\mathbb{C}_{X}\right)$ such that $\mu h \mathrm{hom}\left(\mathcal{G}, \mathcal{O}_{X}\right)$ is concentrated in a single degree. Then the natural morphism

$$
\operatorname{RHom}_{\mathcal{E}_{X}}\left(\mathcal{M}, \operatorname{t\mu hom}\left(\mathcal{G}, \mathcal{O}_{X}\right)\right) \longrightarrow \operatorname{RHom}_{\mathcal{E}_{X}}\left(\mathcal{M}, \mu h o m\left(\mathcal{G}, \mathcal{O}_{X}\right)\right)
$$

is an isomorphism.

Remark 3.5.4. It is slightly complicated to deal with $\mu \mathcal{O}_{X}^{t}$ since we do not know if $\mu \mathcal{O}_{X}^{t}$ is well defined in the derived category of microdifferential modules. The analog of the last proposition should be the formula

$$
\mathrm{RJH}_{\mathcal{H}} \operatorname{mom}_{\beta \mathcal{E}_{X}}\left(\beta \mathcal{M}, \mu \mathcal{O}_{X}\right) \stackrel{\sim}{\longrightarrow} \mathrm{RJH}_{\mathcal{H}} \operatorname{om}_{\beta \mathcal{E}_{X}}\left(\beta \mathcal{M}, \mu \mathcal{O}_{X}^{t}\right)
$$

for any regular holonomic $\mathcal{E}_{X}$-module $\mathcal{M}$. However the second term of this isomorphism is unfortunately not (yet known to be) well-defined. Therefore we only get the following weaker statement:

Proposition 3.5.5. Let $\mathcal{M}$ be a regular holonomic $\mathcal{E}_{X}$-module. Then there is a natural morphism

$$
\mathrm{RJH} \operatorname{com}_{\beta \mathcal{E}_{X}}\left(\beta \mathcal{M}, \mu \mathcal{O}_{X}\right) \longrightarrow \mathrm{RJH} \mathcal{H o m}\left(\beta \mathcal{M}, \mu \mathcal{O}_{X}^{t}\right)
$$

Proof. First we will show that if $\mathcal{F}$ is a microlocal perverse sheaf, then the presheaf

$$
\mathcal{H} \operatorname{com}\left(\mathcal{F}, \operatorname{RJH} \mathcal{H o m}\left(\beta \mathcal{M}, \mu \mathcal{O}_{X}^{t}\right)\right)
$$

is a sheaf. It is sufficient to prove that $\mathrm{R} \mathcal{H} \operatorname{om}\left(\mathcal{F}, \operatorname{RJ\mathcal {H}om}\left(\beta \mathcal{M}, \mu \mathcal{O}_{X}^{t}\right)\right)$ is concentrated in positive degrees. This can be seen by considering

$$
\begin{aligned}
& \operatorname{R\mathcal {H}om}\left(\mathcal{F}, \operatorname{RJ\mathcal {H}om}\left(\beta \mathcal{M}, \mu \mathcal{O}_{X}^{t}\right)\right) \simeq \operatorname{R\mathcal {H}om}\left(\beta \mathcal{M}, \operatorname{RJH} \mathcal{H} \text { om }\left(\mathcal{F}, \mu \mathcal{O}_{X}^{t}\right)\right) \\
& \simeq \operatorname{RHom}\left(\mathcal{M}, \operatorname{R} \mathcal{H} \operatorname{com}\left(\mathcal{F}, \mu \mathcal{O}_{X}^{t}\right)\right)
\end{aligned}
$$

and the fact that, by Lemma 3.6.1 below, $\operatorname{RH} \mathcal{H} o m\left(\mathcal{F}, \mu \mathcal{O}_{X}^{t}\right)$ is concentrated in degre 0 . 
By the comparison theorem if $\mathcal{G} \in \mathrm{D}_{\text {perv }}^{\mathrm{b}}\left(\mathbb{C}_{X}, U\right)$ we have a natural morphism

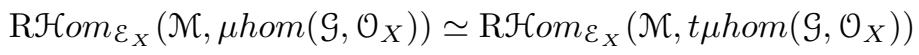

$$
\begin{aligned}
& \longrightarrow \operatorname{RHom}\left(\mathcal{M}, \operatorname{t\mu hom}\left(\mathcal{G}, \mathcal{O}_{X}\right)\right) \text {. }
\end{aligned}
$$

Since we have

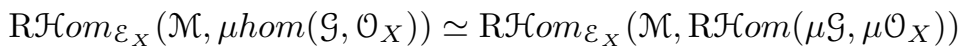

$$
\begin{aligned}
& \simeq \mathrm{RH} \operatorname{com}_{\beta \mathcal{E}_{X}}\left(\beta \mathcal{M}, \operatorname{RJ\mathcal {H}om}\left(\mu \mathcal{G}, \mu \mathcal{O}_{X}\right)\right) \\
& \simeq \mathrm{R} \mathcal{H o m}\left(\mu \mathcal{G}, \operatorname{RJH} \operatorname{Com}_{\beta \mathcal{E}_{X}}\left(\beta \mathcal{M}, \mu \mathcal{O}_{X}\right)\right)
\end{aligned}
$$

and

$$
\begin{aligned}
& \operatorname{RH} \mathcal{H o m}\left(\mathcal{M}, t \mu h o m\left(\mathcal{G}, \mathcal{O}_{X}\right)\right) \simeq \operatorname{R\mathcal {H}} \operatorname{com}\left(\mathcal{M}, \operatorname{RH} \mathcal{H} o m\left(\mu \mathcal{G}, \mu \mathcal{O}_{X}^{t}\right)\right. \\
& \simeq \mathrm{R} \mathcal{H o m}\left(\beta \mathcal{M}, \operatorname{RJ\mathcal {H}om}\left(\mu \mathcal{G}, \mu \mathcal{O}_{X}^{t}\right)\right) \\
& \simeq \operatorname{RHom}\left(\mu \mathcal{G}, \operatorname{RJ\mathcal {H}om}\left(\beta \mathcal{M}, \mu \mathcal{O}_{X}^{t}\right)\right)
\end{aligned}
$$

we get a morphism

$$
\operatorname{RH} \mathcal{H} o m\left(\mu \mathcal{G}, \operatorname{RJH} \operatorname{lom}_{\beta \mathcal{E}_{X}}\left(\beta \mathcal{M}, \mu \mathcal{O}_{X}\right)\right) \longrightarrow \operatorname{RH} \mathcal{H} o m\left(\mu \mathcal{G}, \operatorname{RJH} \mathcal{H} o m\left(\beta \mathcal{M}, \mu \mathcal{O}_{X}^{t}\right)\right) \text {. }
$$

By Lemma 3.6.2 below the complex $\mathrm{RJ} \mathcal{H} m_{\beta \mathcal{E}_{X}}\left(\mathcal{M}, \mu \mathcal{O}_{X}\right)$ is a microlocal perverse sheaf. Hence locally it is of the form $\mu \mathcal{G}$ for some object $\mathcal{G} \in \mathrm{D}_{\text {perv }}^{\mathrm{b}}\left(\mathbb{C}_{X}, U\right)$. Thus locally the identity morphism of $\mathrm{RJH} \mathcal{H o m}_{\beta \mathcal{E}_{X}}\left(\mathcal{M}, \mu \mathcal{O}_{X}\right)$ defines the desired morphism and we may patch it because of the first part of the proof.

Remark 3.5.6. If $\mu \mathcal{O}_{X}^{t}$ was well-defined in the derived category of $\beta \mathcal{E}_{X^{-}}$ modules then the proof of the last proposition would be sufficient to establish the isomorphism

$$
\operatorname{RJH} \operatorname{com}_{\beta \mathcal{E}_{X}}\left(\beta \mathcal{M}, \mu \mathcal{O}_{X}\right) \stackrel{\sim}{\longrightarrow} \mathrm{RJH} \operatorname{com}_{\beta \mathcal{E}_{X}}\left(\beta \mathcal{M}, \mu \mathcal{O}_{X}^{t}\right) .
$$

\section{§3.6. Microlocal Riemann-Hilbert morphism}

In this section we formulate and prove the Microlocal Riemann-Hilbert theorem.

Lemma 3.6.1. Let $\mathcal{F} \in \mu \operatorname{Perv}(\Omega)$ and set $U=\gamma^{-1}(\Omega)$. Then

$$
\operatorname{RH} \mathcal{H} o m\left(\mathcal{F},\left.\mu \mathcal{O}_{X}^{t}\right|_{U}\right)
$$


is an $\left.\mathcal{E}_{X}^{\mathbb{R}, f}\right|_{U}$-module. Moreover

$$
\gamma^{-1} \mathrm{R} \gamma_{*} \mathrm{RHom}\left(\mathcal{F},\left.\mu \mathcal{O}_{X}^{t}\right|_{U}\right)
$$

is a regular holonomic $\left.\mathcal{E}_{X}\right|_{U}$-module.

Proof. First we will show that $\operatorname{R\mathcal {H}} \operatorname{com}\left(\mathcal{F},\left.\mu \mathcal{O}_{X}^{t}\right|_{U}\right)$ is a well-defined $\left.\mathcal{E}_{X}^{\mathbb{R}, f}\right|_{U^{-}}$ module.

By Proposition 3.3.6 it is enough to prove that the complex

$$
\mathrm{RH} \mathcal{H} o m\left(\mathcal{F},\left.\mu \mathcal{O}_{X}^{t}\right|_{U}\right)
$$

is concentrated in a single degree. This is a local problem. Thus we may assume that $\mathcal{F} \simeq \mu \tilde{\mathcal{F}}$ where $\tilde{\mathcal{F}}$ is an object of $\mathrm{D}_{\text {perv }}^{\mathrm{b}}\left(\mathbb{C}_{X}, U\right)$. Therefore we get

$$
\left.\operatorname{RH} \mathcal{H o m}\left(\mathcal{F},\left.\mu \mathcal{O}_{X}^{t}\right|_{U}\right) \simeq \operatorname{R\mathcal {H}om}\left(\left.\mu \widetilde{\mathcal{F}}\right|_{U},\left.\mu \mathcal{O}_{X}^{t}\right|_{U}\right) \simeq \operatorname{thhom}\left(\widetilde{\mathcal{F}}, \mathcal{O}_{X}\right)\right|_{U}
$$

Since $\left.\operatorname{thhom}\left(\tilde{\mathcal{F}}, \mathcal{O}_{X}\right)\right|_{U}$ is invariant under quantized contact transformations, we can suppose that $\widetilde{\mathcal{F}}$ is a perverse sheaf. Then the complex $\left.t \mu h o m\left(\widetilde{\mathcal{F}}, \mathcal{O}_{X}\right)\right|_{U}$ is concentrated in degree 0 . Hence $\operatorname{RHom}\left(\mathcal{F},\left.\mu \mathcal{O}_{X}^{t}\right|_{U}\right)$ is a well-defined $\left.\mathcal{E}_{X}^{\mathbb{R}, f}\right|_{U^{-}}$ module.

Hence $\gamma^{-1} \mathrm{R} \gamma_{*} \operatorname{RHom}\left(\mathcal{F},\left.\mu \mathcal{O}_{X}^{t}\right|_{U}\right)$ is an $\mathcal{E}_{X}$-module. Let us show that it is regular holonomic. This is again a local question, invariant by quantized contact transformations. Therefore we may assume that $\mathcal{F} \simeq \mu \widetilde{\mathcal{F}}$ for a perverse sheaf $\widetilde{\mathcal{F}}$. Recall that

$$
\left.\left.\operatorname{t\mu hom}\left(\tilde{\mathcal{F}}, \mathcal{O}_{X}\right)\right|_{U} \simeq\left(\mathcal{E}_{X}^{\mathbb{R}, f} \underset{\pi^{-1} \mathcal{D}_{X}}{\otimes} \pi^{-1} \operatorname{TH} \mathcal{H o m}\left(\tilde{\mathcal{F}}, \mathcal{O}_{X}\right)\right)\right|_{U}
$$

By the Riemann-Hilbert Theorem $\operatorname{TH} \mathcal{H o m}\left(\tilde{\mathcal{F}}, \mathcal{O}_{X}\right)$ is a regular holonomic $\mathcal{D}_{X^{-}}$ module. Hence the module $\gamma^{-1} \mathrm{R} \gamma_{*} \mathrm{R} \mathcal{H} \operatorname{com}\left(\mathcal{F},\left.\mu \mathcal{O}_{X}^{t}\right|_{U}\right)$ is regular holonomic.

Lemma 3.6.2. Let $\mathcal{M} \in \mathcal{H} \operatorname{Hol} \operatorname{Reg}(U)$ where $U=\gamma^{-1}(\Omega)$. Then

$$
\operatorname{RJH} \operatorname{som}_{\left.\beta \mathcal{E}_{X}\right|_{U}}\left(\beta \mathcal{M},\left.\mu \mathcal{O}_{X}\right|_{U}\right)
$$

is an object of $\mu \operatorname{Perv}(\Omega)$.

Proof. This is a local problem, invariant by quantized contact transformations. Therefore we may assume that $\mathcal{M}$ is isomorphic to $\mathcal{E}_{X} \underset{\pi^{-1} \mathcal{D}_{X}}{\otimes} \pi^{-1} \tilde{\mathcal{M}}$ on $U$ where $\tilde{\mathcal{M}}$ is a regular holonomic $\mathcal{D}_{X}$-module. Then

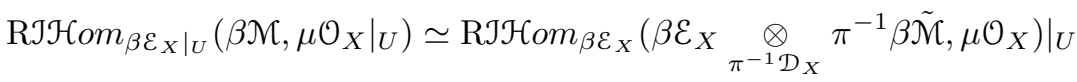

$$
\begin{aligned}
& \left.\simeq \mu \mathrm{R} \mathcal{H} \operatorname{com}_{\mathcal{D}_{X}}\left(\tilde{\mathcal{M}}, \mathcal{O}_{X}\right)\right|_{U} .
\end{aligned}
$$


By the Riemann-Hilbert Theorem $\mathrm{R} \mathcal{H} \operatorname{com}_{\mathcal{D}_{X}}\left(\tilde{\mathcal{K}}, \mathcal{O}_{X}\right)$ is a perverse sheaf.

Definition 3.6.3. $\quad$ For any open subset $\Omega \subset P^{*} X$ let us define the microlocal Riemann-Hilbert correspondence:

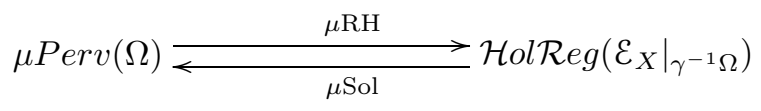

by the formulas

$$
\begin{gathered}
\mu \operatorname{Sol}(\mathcal{M})=\operatorname{RJH} \operatorname{Lom}_{\beta\left(\left.\mathcal{E}_{X}\right|_{\gamma-1}\right)}\left(\beta(\mathcal{M}),\left.\mu \mathcal{O}_{X}\right|_{\gamma^{-1} \Omega}\right) \\
\mu \mathrm{RH}(\mathcal{F})=\gamma_{\Omega}^{-1} \operatorname{R} \gamma_{\Omega *}\left(\mathrm{RHom}\left(\mathcal{F},\left.\mu \mathcal{O}^{t}\right|_{\gamma^{-1}(\Omega)}\right)\right)
\end{gathered}
$$

where $\gamma_{\Omega}$ is the restriction of $\gamma$ to $\gamma^{-1}(\Omega)$.

The functors $\mu \mathrm{Sol}$ and $\mu \mathrm{RH}$ are obviously functors of stacks.

Lemma 3.6.4. There is a natural morphism

$$
\mathrm{Id} \longrightarrow \mu \mathrm{Sol} \circ \mu \mathrm{RH} \text {. }
$$

Proof. Let $\mathcal{F}$ be a microlocal perverse sheaf. We will define the morphism (3.6.1) by a natural element of

$$
\operatorname{Hom}_{\mathrm{D}^{\mathrm{b}}\left(\mathrm{I}\left(\mathbb{C}_{X}\right)\right)}\left(\mathcal{F}, \mathrm{RJH}_{\mathcal{H}} \operatorname{mom}_{\beta \mathcal{E}_{X}}\left(\beta \gamma^{-1} \mathrm{R} \gamma_{*} \operatorname{R} \mathcal{H} \operatorname{com}\left(\mathcal{F}, \mu \mathcal{O}_{X}^{t}\right), \mu \mathcal{O}_{X}\right)\right) .
$$

Note that

$$
\begin{aligned}
\operatorname{Hom}_{\mathrm{D}^{\mathrm{b}}\left(\mathrm{I}\left(\mathbb{C}_{X}\right)\right)}\left(\mathcal{F}, \operatorname{RJH}_{\mathcal{H}} \operatorname{Hom}_{\beta \mathcal{E}_{X}}\left(\beta \gamma^{-1} \mathrm{R} \gamma_{*} \operatorname{R\mathcal {H}} \operatorname{com}\left(\mathcal{F}, \mu \mathcal{O}_{X}^{t}\right), \mu \mathcal{O}_{X}\right)\right) \\
\simeq \operatorname{Hom}_{\mathrm{D}^{\mathrm{b}}\left(\mathrm{I}\left(\beta \mathcal{E}_{X}\right)\right)}\left(\mathcal{F} \otimes \beta \gamma^{-1} \mathrm{R} \gamma_{*} \operatorname{R\mathcal {H}} \operatorname{Com}\left(\mathcal{F}, \mu \mathcal{O}_{X}^{t}\right), \mu \mathcal{O}_{X}\right) .
\end{aligned}
$$

Since the morphism

$$
\beta \gamma^{-1} \mathrm{R} \gamma_{*} \operatorname{RH} \mathcal{H} o m\left(\mathcal{F}, \mu \mathcal{O}_{X}^{t}\right) \longrightarrow \beta \gamma^{-1} \mathrm{R} \gamma_{*} \operatorname{R} \mathcal{H} \operatorname{com}\left(\mathcal{F}, \mu \mathcal{O}_{X}\right)
$$

is $\beta \mathcal{E}_{X}$-linear, the natural morphism in $\mathrm{D}^{\mathrm{b}}\left(\mathrm{I}\left(\beta \mathcal{E}_{X}\right)\right)$

$$
\mathcal{F} \otimes \beta \gamma^{-1} \mathrm{R} \gamma_{*} \mathrm{R} \mathcal{H} \operatorname{com}\left(\mathcal{F}, \mu \mathcal{O}_{X}\right) \longrightarrow \mu \mathcal{O}_{X}
$$

defines the morphism of the lemma.

Now we are ready to prove the microlocal Riemann-Hilbert Theorem: 
Theorem 3.6.5. The functors $\mu \mathrm{Sol}$ and $\mu \mathrm{RH}$ define quasi-inverse equivalences of stacks

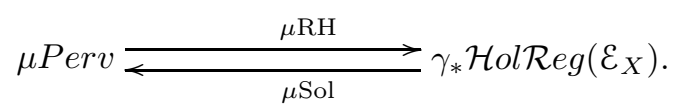

Proof. First let us show that the morphism of Lemma 3.6.4 is an isomorphism.

Let $\mathcal{F}$ be a microlocal perverse sheaf defined in a neighborhood at $p$. Then there exists $\widetilde{\mathcal{F}} \in \mathrm{D}_{\text {perv }}^{\mathrm{b}}\left(\mathbb{C}_{X}, \mathbb{C}^{\times} p\right)$ such that $\mathcal{F} \simeq \mu \widetilde{\mathcal{F}}$. Let $\chi$ be a contact transformation such that $\chi(\operatorname{SS}(\widetilde{\mathcal{F}}))$ is in generic position at $\chi(p)$. Then

$$
\begin{aligned}
& \Phi_{\mu \mathcal{K}}^{a} \operatorname{RJ\mathcal {H}} \operatorname{Hom}_{\beta \varepsilon_{X}}\left(\beta \operatorname{RH} \mathcal{H} o m\left(\mu \widetilde{\mathcal{F}}, \mu \mathcal{O}_{X}^{t},\right), \mu \mathcal{O}_{X}\right) \\
& \simeq \operatorname{RJ\mathcal {H}} \operatorname{Hom}_{\beta \mathcal{E}_{X}}\left(\beta \operatorname{R\mathcal {H}} \operatorname{com}\left(\Phi_{\mathcal{K}} \widetilde{\mathcal{F}}, \mu \mathcal{O}_{X}^{t},\right), \mu \mathcal{O}_{X}\right) .
\end{aligned}
$$

Since $\Phi_{\mathcal{K}} \widetilde{\mathcal{F}}$ is isomorphic to a perverse sheaf in a neighborhood of $\pi(p)$ the isomorphism follows from the second part of Proposition 3.5.2.

Now let us show that the functor $\mu \mathrm{RH}$ is an equivalence of stacks. It is sufficient to prove this locally. In order to prove that $\mu \mathrm{RH}$ is essentially surjective, we show that $\mu \mathrm{RH}$ and $\mu \mathrm{Sol}$ are inverse to each other (up to isomorphism) on the level of objects. We have already seen that $\mu \operatorname{Sol} \circ \mu \mathrm{RH}(\mathcal{F}) \simeq \mathcal{F}$ for any microlocal perverse sheaf $\mathcal{F}$.

Let $\mathcal{M}$ be a regular holonomic $\mathcal{E}_{X}$-module defined in a neighborhood of $p \in \dot{T}^{*} X$. Let $\chi$ be a contact transformation such that $\chi_{*} \mathcal{M}$ is in generic position at $\chi(p)$. Then

$$
\begin{aligned}
& \chi_{*} \mathrm{R} \gamma^{-1} \mathrm{R} \gamma_{*} \mathrm{R} \mathcal{H} \text { om }\left(\mathrm{RJ \mathcal {H }} \operatorname{com}_{\beta \mathcal{E}_{X}}\left(\beta(\mathcal{M}), \mu \mathcal{O}_{X}\right), \mu \mathcal{O}_{X}^{t}\right) \\
& \simeq \gamma^{-1} \mathrm{R} \gamma_{*} \operatorname{RHom}\left(\Phi_{\mu \mathcal{K}}^{a} \operatorname{RJH}_{\mathcal{H}} \operatorname{mom}_{\beta \mathcal{E}_{X}}\left(\beta(\mathcal{M}), \mu \mathcal{O}_{X}\right), \mu \mathcal{O}_{X}^{t}\right) \\
& \simeq \gamma^{-1} \mathrm{R} \gamma_{*} \mathrm{R} \mathcal{H} \text { om }\left(\mathrm{RJH} \operatorname{Hom}_{\beta \mathcal{E}_{X}}\left(\beta \chi_{*} \mathcal{M}, \mu \mathcal{O}_{X}\right), \mu \mathcal{O}_{X}^{t}\right)
\end{aligned}
$$

But since $\chi_{*} \mathcal{M}$ is in generic position there exists a regular holonomic $\mathcal{D}_{X^{-}}$ module $\widetilde{\mathcal{M}}$ such that

$$
\mathcal{M} \simeq \mathcal{E}_{X} \underset{\pi^{-1} \mathcal{D}_{X}}{\stackrel{\mathrm{L}}{\otimes}} \pi^{-1} \tilde{\mathcal{M}}
$$

and we find that $\mu \mathrm{RH} \circ \mu \operatorname{Sol}(\mathcal{M}) \simeq \mathcal{M}$ by the classical Riemann-Hilbert Theorem. Hence $\mu \mathrm{RH}$ is essentially surjective. 
Let us show that $\mu \mathrm{RH}$ is fully faithful. Let $\mathcal{F}, \mathcal{G}$ be microlocal perverse sheaves. By invariance under quantized contact transformations we may assume that there exist perverse sheaves $\widetilde{\mathcal{F}}, \widetilde{\mathcal{G}}$ such that $\mathcal{F} \simeq \mu \widetilde{\mathcal{F}}$ and $\mathcal{G} \simeq \mu \widetilde{\mathcal{G}}$. Then the fact that $\mu \mathrm{RH}$ is fully faithful follows from the well-known formula

$$
\mu h o m(\widetilde{\mathcal{F}}, \widetilde{\mathcal{G}}) \simeq \operatorname{RH} \operatorname{com}(\mathrm{RH}(\widetilde{\mathcal{G}}), \operatorname{RH}(\widetilde{\mathcal{F}}))
$$

\section{A. Appendix - The Functor of Ind-microlocalization}

In this appendix, we first recall some definitions and statements of the theory of analytic ind-sheaves from [KS2]. Then we define Kashiwara's functor of ind-microlocalization and give (without proof) some basic properties that we used in this paper. The main result is the microlocal composition formula (Theorem A.2.5) which is the most important tool for our constructions.

\section{A.1. Ind-sheaves}

If $\mathcal{C}$ is a category, one embeds $\mathcal{C}$ into the category of presheaves (of sets) on $\mathcal{C}$ by the fully faithful Yoneda-functor:

$$
\mathcal{C} \longrightarrow \widehat{\mathcal{C}} ; \quad A \mapsto \operatorname{Hom}_{\mathcal{C}}(\cdot, A)
$$

where $\widehat{\mathcal{C}}$ is the category of contravariant functors $\mathcal{C} \rightarrow \mathcal{S e t}$. An object in the essential image of the Yoneda-functor is called representable. Note that $\widehat{\mathcal{C}}$ admits all small colimits since the category $\mathcal{S}$ et does but even if $\mathcal{C}$ admits colimits the Yoneda-functor does not commute with them.

One denotes by Ind $\mathcal{C}$ the full subcategory of $\widehat{\mathcal{C}}$ formed by small filtered colimits of representable objects and calls it the category of ind-objects of $\mathcal{C}$. Then Ind $\mathcal{C}$ admits all small filtered colimits.

If $\mathcal{C}$ is abelian then $\operatorname{Ind} \mathcal{C}$ is abelian and the Yoneda-functor induces an exact fully faithful functor $\mathcal{C} \rightarrow \operatorname{Ind} \mathcal{C}$.

Now let $X$ be a locally compact topological space with a countable base of open sets and fix a field $k$. One sets

$$
\mathrm{I}\left(k_{X}\right)=\operatorname{Ind} \operatorname{Mod}^{c}\left(k_{X}\right)
$$

where $\mathcal{M o d}^{c}\left(k_{X}\right)$ denotes the full subcategory of $\mathcal{M o d}\left(k_{X}\right)$ formed by sheaves with compact support. We call $\mathrm{I}\left(k_{X}\right)$ the category of ind-sheaves (of $k$-vector 
spaces). One can show that the prestack $X \supset U \mapsto \mathrm{I}\left(k_{U}\right)$ is a proper stack (in the sens of [KS2]), in particular it is an abelian stack.

There are three important basic functors for ind-sheaves

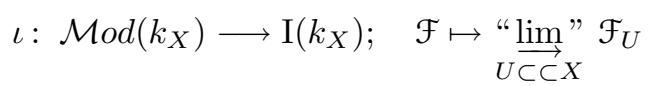

$$
\begin{aligned}
& \alpha: \mathrm{I}\left(k_{X}\right) \longrightarrow \operatorname{Mod}\left(k_{X}\right) ; \quad “ \underset{i \in I}{\lim } " \mathcal{F}_{i} \mapsto \underset{i \in I}{\lim _{i \in I}} \mathcal{F}_{i} \\
& \beta: \operatorname{Mod}\left(k_{X}\right) \longrightarrow \mathrm{I}\left(k_{X}\right) \quad \text { left adjoint to } \alpha
\end{aligned}
$$

where we write "lim" for colimits in the category $\mathrm{I}\left(k_{X}\right)$. All three functors induce functors of stacks.

\section{Proposition A.1.1.}

(i) The functor $\iota$ is fully faithful and exact.

(ii) The functor $\alpha$ is exact.

(iii) The functor $\beta$ is fully faithful and exact.

(iv) The triple $(\beta, \alpha, \iota)$ is a triple of adjoint functors, i.e. $\beta$ is left adjoint to $\alpha$ and $\alpha$ is left adjoint to $\iota$.

Note that since the functors $\iota, \alpha, \beta$ are exact they are well-defined in the derived categories, guard the adjoint properties and $\beta, \iota$ are still fully faithful. An object $\mathcal{F} \in \mathrm{D}^{\mathrm{b}}\left(k_{X}\right)$ is identified with $\iota \mathcal{F}$ in $\mathrm{D}^{\mathrm{b}}\left(\mathrm{I}\left(k_{X}\right)\right)$.

There are internal operations on ind-sheaves

$$
(\cdot) \otimes(\cdot) \quad \text { and } \quad \mathcal{J H o m}(\cdot, \cdot)
$$

and an external

$$
\mathcal{H} \operatorname{com}(\cdot, \cdot): \mathrm{I}\left(k_{X}\right) \times \mathrm{I}\left(k_{X}\right) \longrightarrow \mathcal{M} \text { od }\left(k_{X}\right) .
$$

Moreover for any continuous map $f: X \rightarrow Y$ between locally compact spaces we get the external operations

$$
f^{-1}, f_{*}, f_{! !}
$$

where the notation $f_{! !}$indicates that $\iota f_{!} \not f_{! !} \iota$.

While $\otimes$ and $f^{-1}$ are exact the other functors have a right derived functor and pass to the derived category where we can define Poincaré-Verdier duality, i.e. we have a right adjoint $f^{!}$to $\mathrm{R} f$ !! and we get the usual formalism of Grothendieck's six operations. We will not recall here the various natural isomorphisms relating these functors and refer to [KS2] but let us summarize the commutation properties with $\iota, \alpha, \beta$ : 


\section{Proposition A.1.2.}

(i) The functor $\iota$ commutes to $\otimes, f^{-1}, f^{!}, \mathrm{R} f_{*}$.

(ii) The functor $\alpha$ commutes to $\otimes, f^{-1}, \mathrm{R} f_{*}, \mathrm{R} f_{\text {!! }}$ and we have $\alpha \mathrm{RJ \mathcal {H } o m}(\cdot, \cdot) \simeq$ $\mathrm{R} \mathcal{H o m}(\cdot, \cdot)$.

(iii) The functor $\beta$ commutes to $\otimes, f^{-1}$.

Finally let us state the following Proposition which has no counterpart in classical sheaf theory:

Proposition A.1.3. Let $\mathcal{F}, \mathcal{G} \in \mathrm{D}^{\mathrm{b}}\left(k_{X}\right)$ and $\mathcal{M} \in \mathrm{D}^{\mathrm{b}}\left(\mathrm{I}\left(k_{X}\right)\right)$. Then there is a natural isomorphism

$$
\operatorname{RJH} \mathcal{H o m}(\mathcal{F}, \mathcal{M}) \otimes \beta \mathcal{G} \stackrel{\sim}{\longrightarrow} \operatorname{RJH} \mathcal{H} o m(\mathcal{F}, \mathcal{M} \otimes \beta \mathcal{G}) .
$$

\section{A.2. Microlocalization of ind-sheaves}

In [K5], Kashiwara establishes the following theorem

Theorem A.2.1. $\quad$ There exists a functor

$$
\mu: \mathrm{D}^{\mathrm{b}}\left(\mathrm{I}\left(k_{X}\right)\right) \longrightarrow \mathrm{D}^{\mathrm{b}}\left(\mathrm{I}\left(k_{T^{*} X}\right)\right)
$$

such that for any $\mathcal{F}, \mathcal{G} \in \mathrm{D}^{\mathrm{b}}\left(k_{X}\right)$ we have a natural isomorphism

$$
\operatorname{RHom}(\mu \mathcal{F}, \mu \mathcal{G}) \simeq \operatorname{RHom}\left(\pi^{-1} \mathcal{F}, \mu \mathcal{G}\right) \simeq \mu h o m(\mathcal{F}, \mathcal{G})
$$

Remark A.2.2. Note that if $\mathcal{F} \in \mathrm{D}^{\mathrm{b}}\left(k_{X}\right)$, then

$$
\operatorname{supp}(\mu \mathcal{F})=\operatorname{supp}(\operatorname{R\mathcal {H}om}(\mu \mathcal{F}, \mu \mathcal{F}))=\operatorname{supp}(\mu h o m(\mathcal{F}, \mathcal{F}))=\operatorname{SS}(\mathcal{F})
$$

We recall here the construction of $\mu$ which is rather straight-forward if we want to have the property of the Theorem (cf. Proposition 3.1.4).

The normal deformation of the diagonal in $T^{*} X \times T^{*} X$ can be visualized by the following diagram

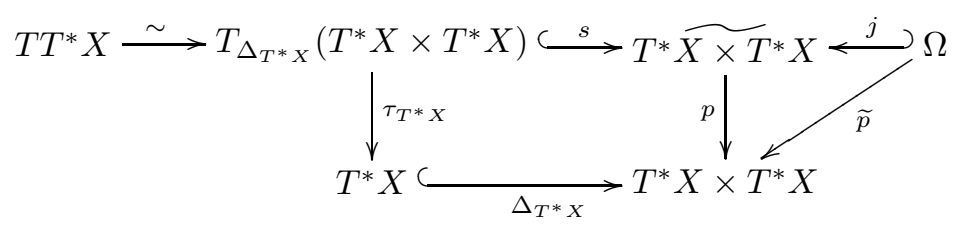


Note that $\tilde{p}$ is smooth but $p$ is not. Also, the square is not cartesian. Set

$$
\mathrm{K}_{X}=\mathrm{R} p_{! !}\left(k_{\bar{\Omega}} \otimes \beta\left(k_{P}\right)\right) \otimes \beta\left(\omega_{\Delta_{T^{*} X} \mid T^{*} X \times T^{*} X}^{\otimes-1}\right)
$$

where the set $P \subset T T^{*} X$ is defined by

$$
P=\left\{\left(x, \xi ; v_{x}, v_{\xi}\right) \mid\left\langle v_{x}, \xi\right\rangle \geqslant 0\right\} .
$$

Definition A.2.3. Kashiwara's functor of microlocalization is defined on $T^{*} X$ as

$$
\mu: \mathrm{D}^{\mathrm{b}}\left(\mathrm{I}\left(k_{X}\right)\right) \longrightarrow \mathrm{D}^{\mathrm{b}}\left(\mathrm{I}\left(k_{T^{*} X}\right)\right) ; \mathcal{F} \mapsto \mu \mathcal{F}=\mathrm{K}_{X} \circ \pi^{-1} \mathcal{F} .
$$

We made use of the following proposition.

Proposition A.2.4. Let $\mathcal{F} \in \mathrm{D}_{\mathbb{R}-c}^{\mathrm{b}}\left(k_{X}\right)$ and $\mathcal{G} \in \mathrm{D}^{\mathrm{b}}\left(\mathrm{I}\left(k_{X}\right)\right)$ and assume that

$$
\operatorname{SS}(\mathcal{F}) \cap \operatorname{supp}(\mu \mathcal{G}) \subset T_{X}^{*} X
$$

Then there is a natural isomorphism

$$
\operatorname{RH} \mathcal{H o m}\left(\mathcal{F}, k_{X}\right) \otimes \mathcal{G} \stackrel{\sim}{\longrightarrow} \operatorname{RJH} \mathcal{H o m}(\mathcal{F}, \mathcal{G})
$$

The main theorem of [K5] is the microlocal composition theorem.

Theorem A.2.5. Microlocal composition of kernels. Let $\mathcal{K}_{1} \in \mathrm{D}^{\mathrm{b}}$ $\left(\mathrm{I}\left(k_{X \times Y}\right)\right)$ and $\mathcal{K}_{2} \in \mathrm{D}^{\mathrm{b}}\left(\mathrm{I}\left(k_{Y \times Z}\right)\right)$.

(1) There is a natural morphism

$$
\mu_{X \times Y} \mathcal{K}_{1} \stackrel{a}{\circ} \mu_{Y \times Z} \mathcal{K}_{2} \longrightarrow \mu_{X \times Z}\left(\mathcal{K}_{1} \circ \mathcal{K}_{2}\right) .
$$

(2) Assume the non-characteristic condition ${ }^{9}$

$$
\mathrm{SS}_{0}\left(\mathcal{K}_{1}\right) \underset{T^{*} Y}{\stackrel{a}{\times}} \operatorname{SS}_{0}\left(\mathcal{K}_{2}\right) \cap\left(T_{X}^{*} X \times T^{*} Y \times T_{Z}^{*} Z\right) \subset T_{X}^{*} X \times T_{Y}^{*} Y \times T_{Z}^{*} Z,
$$

\footnotetext{
${ }^{9}$ For two sets $S_{1} \subset T^{*} X \times T^{*} Y$ and $S_{2} \subset T^{*} Y \times T^{*} Z$ we denote by $S_{1} \underset{T^{*} Y}{\stackrel{a}{×}} S_{2}$ the cartesian product of $\left.q_{2}^{a}\right|_{S_{1}}: S_{1} \rightarrow T^{*} Y$ and $\left.q_{1}\right|_{S_{2}}: S_{2} \rightarrow T^{*} Y$, hence

$$
\begin{aligned}
S_{1} \underset{T^{*} Y}{\stackrel{a}{×}} S_{2}=\{ & \left(\left(x ; \xi_{x}\right),\left(y ; \xi_{Y}\right),\left(z ; \xi_{Z}\right) \in T^{*} X \times T^{*} Y \times T^{*} Z \mid\right. \\
& \left.\left(\left(x ; \xi_{x}\right),\left(y ; \xi_{Y}\right)\right) \in S_{1} \quad\left(\left(y ;-\xi_{Y}\right),\left(z ; \xi_{Z}\right)\right) \in S_{2}\right\} .
\end{aligned}
$$
}


then the morphism

$$
\mathrm{K}_{X \times Z} \circ\left(\mu_{X \times Y} \mathcal{K}_{1} \stackrel{a}{\circ} \mu_{Y \times Z} \mathcal{K}_{2}\right) \longrightarrow \mu_{X \times Z}\left(\mathcal{K}_{1} \circ \mathcal{K}_{2}\right)
$$

is an isomorphism and

$$
\begin{aligned}
& \mu_{X \times Y} \mathcal{K}_{1} \stackrel{a}{\circ} \mu_{Y \times Z} \mathcal{K}_{2} \longrightarrow \mu_{X \times Z}\left(\mathcal{K}_{1} \circ \mathcal{K}_{2}\right)
\end{aligned}
$$

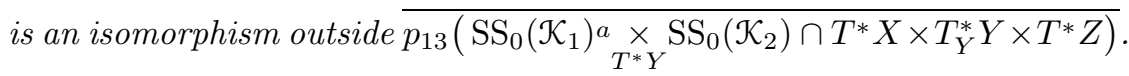

\section{References}

[An1] Andronikof, E., A microlocal version of the Riemann-Hilbert correspondence, Topol. Methods Nonlinear Anal., 4 (1994).

[An2] Microlocalisation tempérée, Bull. Soc. Math. France, 122 (1994).

[BBD] Beilinson, B., Bernstein, J. and Deligne, P., Faisceaux pervers, Analyse et Topologie sur les Espaces Singuliers, Astérisque 100, (1982), 1-171.

[GMV] Gelfand, S., MacPherson R. and Vilonen, K. Microlocal Perverse Sheaves, anounced.

[K1] Kashiwara, M., Systems of microdifferential equations, Progr. Math., 34 Birkhæuser, (1983).

[K2] The Riemann-Hilbert Problem for Holonomic Systems, Publ. RIMS, Kyoto Univ., 20 (1984), 319-365.

[K3] - Introduction to microlocal analysis, Enseign. Math., Monogr., 32 (1986).

[K4] - Quantization of Contact Manifolds, Publ. RIMS, Kyoto Univ., 32 (1996).

[K5] — Ind-Microlocalization, written by F. Ivorra and I. Waschkies following a manuscript of M. Kashiwara, to appear in Prog. Math., Birkhäuser.

[KK] Kashiwara, M. and Kawai, T., On Holonomic Systems of Microdifferential Equations III, Publ. RIMS, Kyoto Univ., 17 (1981).

[KS1] Kashiwara, M. and Schapira, P., Sheaves on Manifolds, Springer, 1990.

[KS2] Ind-Sheaves, Astérisque 271, (2001).

[Sch] Schapira, P., Microdifferential Systems in the Complex Domain, Grundlehren Math. Wiss. 269, Springer, 1985.

[SKK] Sato, M., Kawai, T. and Kashiwara, M., Hyperfunctions and pseudo-differential equations, Proceedings Katata 1971, H. Komatsu (ed.), Lecture Notes in Math., 287, Springer, 1973, pp. 265-529.

[W] Waschkies, I., The Stack of Microlocal Perverse Sheaves, Bull. Soc. Math. France, 132 (2004), 397-462. 